\title{
Multivalent Lactose-Ferrocene Conjugates Based on Poly (Amido Amine) Dendrimers and Gold Nanoparticles as Electrochemical Probes for Sensing Galectin-3
}

\author{
Manuel C. Martos-Maldonado, Indalecio Quesada-Soriano, Luis García-Fuentes and \\ Antonio Vargas-Berenguel * (1) \\ Department of Chemistry and Physics, University of Almería, Carretera de Sacramento s/n, 04120 Almería, \\ Spain; mmm588@inlumine.ual.es (M.C.M.-M.); indaqs@ual.es (I.Q.-S.); lgarcia@ual.es (L.G.-F.) \\ * Correspondence: avargas@ual.es
}

Received: 26 December 2019; Accepted: 19 January 2020; Published: 24 January 2020

\begin{abstract}
Galectin-3 is considered a cancer biomarker and bioindicator of fibrosis and cardiac remodeling and, therefore, it is desirable to develop convenient methods for its detection. Herein, an approach based on the development of multivalent electrochemical probes with high galectin-3 sensing abilities is reported. The probes consist of multivalent presentations of lactose-ferrocene conjugates scaffolded on poly (amido amine) (PAMAM) dendrimers and gold nanoparticles. Such multivalent lactose-ferrocene conjugates are synthesized by coupling of azidomethyl ferrocene-lactose building blocks on alkyne-functionalized PAMAM, for the case of the glycodendrimers, and to disulfide-functionalized linkers that are then used for the surface modification of citrate-stabilized gold nanoparticles. The binding and sensing abilities toward galectin-3 of both ferrocene-containing lactose dendrimers and gold nanoparticles have been evaluated by means of isothermal titration calorimetry, UV-vis spectroscopy, and differential pulse voltammetry. The highest sensitivity by electrochemical methods to galectin-3 was shown by lactosylferrocenylated gold nanoparticles, which are able to detect the lectin in nanomolar concentrations.
\end{abstract}

Keywords: galectin-3; electrochemical probes; gold nanoparticles; ferrocene; electroactive glycodendrimers; PAMAM

\section{Introduction}

Galectins are a family of $\beta$-D-galactoside-binding lectins, which are widespread throughout the animal kingdom and are ubiquitous in adult humans. Among these lectins, galectin-3 (Gal-3) occupies a prominent place due to its implications in various physiological and pathological processes, such as inflammation, cancer progression, and metastasis through various mechanisms, including cell-cell adhesion, cell-matrix interactions, apoptosis, angiogenesis, and mRNA splicing. Gal-3 plays a key role in triggering inflammation in many acute and chronic conditions, including atherosclerosis and heart failure, autoimmune diseases, neurological degeneration, and diabetes, among others. Gal-3 is overexpressed in many tumor cells and is considered to be a cancer biomarker [1]. Furthermore, high blood Gal-3 levels have been correlated with the likelihood of heart failure, and the protein is a novel bioindicator of fibrosis and cardiac remodeling [2-7]. Therefore, there is a need for the development of Gal-3 detection and monitoring methods in serum to provide useful information on cancer progression and cardiovascular risk. Such methods should meet the characteristics of convenience, inexpensiveness, and robustness. 
Most of the reported assay methods are based on routine immunoassays [8,9], Western blot analysis or an enzyme-linked immunosorbent assay (ELISA) [10-12], and immunohistochemical methods [13,14]. However, despite the advantages of immunoassay-type methods, they exhibit many inherent limitations; they can be labor-intensive, expensive, and suffer from a lack of reproducibility, a high possibility of false positive/negative results, and a lack of stability of antibodies and enzymes $[15,16]$.

To improve Gal-3 detection sensitivity and reproducibility, immunoassay-type methods can be combined with other techniques. For example, a sandwich-type electrochemical immunosensor that uses Gal-3 antibodies has been reported recently [17]. This method is based on MIL-88(Fe) metal-organic framework/Au hybrid nanoparticles immobilized on $\mathrm{N}$-doped graphene nanoribbons for the immobilization of the primary Gal-3 antibody and involves AuPt-methylene blue. The latter is a redox nanoprobe for the generation and amplification of electrochemical signals. Although direct detection of Gal-3 by means of surface plasmon resonance (SPR) measurements using lactose-modified gold surfaces has been reported [18], an optical biosensor that uses the anti-Gal-3 antibody as a biorecognition element has been developed for the quantification of Gal-3 based on a graphene oxide-enhanced SPR methodology for transducing the immunological interaction [19].

Efforts to develop alternative sensing methods include the use of galactose-containing chemical probes. A biotinylated $\mathrm{N}$-acetyl lactosamine-polyacrylamide conjugate has been used as a probe for Gal-3 sensing in a sandwich-type assay. The immobilized lectin bound to the conjugate is detected by means of the enhanced chemiluminescence of horseradish peroxidase-streptavidin bound to the probe [20].

Synthesized molecular probes with an enhanced affinity to Gal-3 have been applied in optical Gal-3 sensing. This method consists of using ligands, such as lactose or thiodigalactoside derivatives bearing a photo label for covalent attachment to Gal-3 upon binding and photoirradiation, and an azido or propargyl group for the subsequent "clicking" of fluorescent derivatives for in-gel visualization of the protein [21-24].

Furthermore, the synthesis of a lactose derivative containing a lanthanide-binding tag allows for the use of such ligands as a molecular probe for sensing Gal-3 through NMR measurements, thereby exploiting the paramagnetic effects of the presence of the lanthanide cation [25].

However, the development of electrochemical probes for the detection of Gal-3 is very scarce. So far, to the best of our knowledge, only two reported examples involve the use of electrochemical methods $[17,26]$. Of them, only one is based on the use of a Gal-3 ligand probe; that is, galactose-containing single-walled carbon nanotubes [26].

Electrochemical sensors have found a wide range of applications in industrial, environmental, clinical, agricultural, and food analysis. Such devices present some advantages. For example, they can be used for direct measurements in complex matrices, they are susceptible to miniaturization, and they can provide rapid, highly sensitive, reliable, and inexpensive measurements [27-30].

We previously reported on the redox and sensing ability properties of carbohydrate and peptide-ferrocene conjugates toward proteins [31-39]. An interesting finding was the fact that the multivalent presentation of ferrocene-mannose conjugates led to a remarkable enhancement of the protein sensing properties due to the multivalent glycoside effect and the multielectron exchange as a result of the multiple ferrocene units [36,38]. In addition, the electroactive mannosylated gold nanoparticles complemented their redox sensing abilities with the optical sensing abilities that derive from their plasmonic properties. In this paper, we use our approach to develop multivalent electrochemical probes with high Gal-3 sensing abilities. Since many lectins are oligomeric, a very successful strategy to obtain high-affinity ligands is the design of multivalent compounds. Nevertheless, Gal-3 is mainly present in solution in a monomeric form in rapid equilibrium with a small percentage of protein oligomer [40]. The Gal-3 structure comprises, in addition to the carbohydrate recognition domain (CRD), an N-terminal, and collagen-like domains that have demonstrated to be important for the formation of Gal-3 oligomers, though CRD is also claimed to be involved in inducing aggregation [4,40-42]. For example, the specific interaction of Gal-3 with multivalent carbohydrates 
induces the formation of cross-linked complexes involving Gal-3 pentamers [40]. In addition, dynamic light scattering (DLS) experiments have shown that lactose-functionalized poly (amido amine) (PAMAM) dendrimers of generation 2 to 6 induce Gal-3 aggregation [43]. Moreover, a multivalent effect was reported to arise from the enhanced binding affinity of glycoclusters bearing thiodigalactoside derivatives toward Gal-3 [44].

Our studies started with the synthesis of electroactive poly (amidoamine) (PAMAM)-based dendrimers from generation 0 to 2 , as well as gold nanoparticles bearing lactosylferrocenyl moieties in the periphery. The binding and sensing abilities of such multivalent systems toward Gal-3 have been evaluated by means of isothermal titration calorimetry, UV-vis spectroscopy, and differential pulse voltammetry.

\section{Materials and Methods}

\subsection{General Methods}

Thin-layer chromatography (TLC) was performed on Merck silica gel 60 F254 aluminum sheets and developed by UV-vis light and ethanolic sulfuric acid ( $5 \% v / v)$. Flash column chromatography was performed on Merck silica gel (230-400 mesh, ASTM, West Conshohocken, PA, USA). Infrared spectra were recorded on a Bruker Alpha FTIR equipped with a Bruker universal ATR sampling accessory. ${ }^{1} \mathrm{H}$ and ${ }^{13} \mathrm{C}$ NMR spectra were recorded on Bruker Avance DPX300 and Bruker Avance 500 Ultrashield spectrometers equipped with $\mathrm{QNP}{ }^{1} \mathrm{H} /{ }^{13} \mathrm{C} /{ }^{19} \mathrm{~F} /{ }^{31} \mathrm{P}$ and inverse TBI ${ }^{1} \mathrm{H} /{ }^{31} \mathrm{P} / \mathrm{BB}$ probes, respectively. Standard Bruker software was used for acquisition and processing routines. Chemical shifts are given in parts per million (ppm) and referenced to internal TMS $\left(\delta_{\mathrm{H}}, \delta_{\mathrm{C}} 0.00\right)$. $J$ values are given in hertz (Hz). MALDI-TOF mass spectra were recorded on a 4800 Plus AB SCIEX spectrometer with 2,5-dihydroxybenzoic acid (DHB) as the matrix. ESI-TOF mass spectra were measured on a Waters Xevo Qtof spectrometer. All aqueous procedures used pure water (Milli-Q, $18.2 \mathrm{M} \Omega \mathrm{cm}$ ) obtained from a Millipore Milli-Q Plus system. Centrifugal filtrations were carried out on a Digicen 21R centrifuge using Amicon Millipore $10 \mathrm{kDa}$ MWCO and $3 \mathrm{kDa}$ MWCO centrifugal filters for purification and concentration purposes, respectively. Transmission electron microscopy (TEM) analyses were carried out on a Carl Zeiss LIBRA 120 PLUS instrument at $120 \mathrm{keV}$. All reagents were purchased from Sigma Aldrich and used without further purification. Compounds 2 [36], 3 [31,32], 15 [36], and 16 [36] were synthesized as previously reported. Human galectin-3 was expressed and purified as previously reported [45].

\subsection{Synthesis}

2.2.1.

1-(Hydroxymethyl)-1'-(\{4-[4-O-( $\beta$-D-Galactopyranosyl)- $\beta$-D-glucopyranosyloxymethyl]-1H-1,2,3-triazol-1-yl\} methyl)ferrocene (5)

$\mathrm{CuSO}_{4}(40 \mathrm{mg}, 0.183 \mathrm{mmol})$ and sodium ascorbate $(160 \mathrm{mg}, 1.003 \mathrm{mmol})$ were added to a stirred solution of compounds 3 (400 mg, $1.476 \mathrm{mmol})$ and 4 (465 mg, $1.222 \mathrm{mmol})$ in $\mathrm{THF} / \mathrm{H}_{2} \mathrm{O}(24 \mathrm{~mL}, 1: 1)$ under $\mathrm{N}_{2}$. The resulting mixture was stirred at room temperature for $16 \mathrm{~h}$ and then $-10 \%$ aqueous $\mathrm{NH}_{3}$ was added $(20 \mathrm{~mL})$. The resulting solution was filtered through silica gel. After that, the solvent was evaporated to dryness at reduced pressure avoiding heating over $40^{\circ} \mathrm{C}$. The crude was purified by column chromatography on silica gel (EtOAc/ $\mathrm{MeOH}, 2: 1)$ to obtain compound 5 (790 mg, $1.213 \mathrm{mmol}$, $99 \%)$ as a yellow solid. IR $\left(\mathrm{KBr}, \mathrm{cm}^{-1}\right) 3373,2925,2882,1665,1401,1380,1331,1236,1156,1116,1051$, 921, 893, 511, 486; ${ }^{1} \mathrm{H}$ NMR (300 MHz; CD $\left.{ }_{3} \mathrm{OD}\right) 7.97$ (s, 1H, H-5-C2HN3), 5.22 (s, 2H, CH2N3), 4.96 (d, $1 \mathrm{H}, 2 J=12.4 \mathrm{~Hz}, \mathrm{CHO}), 4.82(\mathrm{~d}, 1 \mathrm{H}, 2 \mathrm{~J}=12.4 \mathrm{~Hz}, \mathrm{CHO}), 4.44\left(\mathrm{~d}, 1 \mathrm{H}, 3 J_{1,2}=7.6 \mathrm{~Hz}, \mathrm{H}-1\right), 4.41-4.36$ $\left(\mathrm{m}, 3 \mathrm{H}, \mathrm{H}-1^{\prime}, \mathrm{CpCH}_{2} \mathrm{OH}\right), 4.34\left(\mathrm{t}, 2 \mathrm{H},{ }^{3} \mathrm{~J}=1.7 \mathrm{~Hz}, \mathrm{H}_{\mathrm{Cp}}\right), 4.28\left(\mathrm{t}, 2 \mathrm{H},{ }^{3} \mathrm{~J}=1.7 \mathrm{~Hz}, \mathrm{H}_{\mathrm{Cp}}\right), 4.23\left(\mathrm{t}, 2 \mathrm{H},{ }^{3} \mathrm{~J}=\right.$ $\left.1.7 \mathrm{~Hz}, \mathrm{H}_{\mathrm{Cp}}\right), 4.21\left(\mathrm{t}, 2 \mathrm{H},{ }^{3} \mathrm{~J}=1.7 \mathrm{~Hz}, \mathrm{HCp}\right), 3.95\left(\mathrm{~d}, 1 \mathrm{H},{ }^{2} J_{6,6^{\prime}}=11.4 \mathrm{~Hz}, \mathrm{H}-6\right), 3.91-3.83(\mathrm{~m}, 2 \mathrm{H}, \mathrm{H}-6$, $\left.4^{\prime}\right)$, 3.82-3.67 (m, 3H, H-6' $\left.6^{\prime}, 5^{\prime}\right), 3.66-3.51\left(\mathrm{~m}, 3 \mathrm{H}, \mathrm{H}-4,3,2^{\prime}\right), 3.50-3.43\left(\mathrm{~m}, 1 \mathrm{H}, \mathrm{H}-3^{\prime}\right), 3.40-3.37$ (m, $1 \mathrm{H}, \mathrm{H}-5), 3.32-3.27(\mathrm{~m}, 1 \mathrm{H}, \mathrm{H}-2) ;{ }^{13} \mathrm{C}$ NMR $\left(75 \mathrm{MHz} ; \mathrm{CD}_{3} \mathrm{OD}\right) 145.5\left(\mathrm{C}-4-\mathrm{C}_{2} \mathrm{HN}_{3}\right), 124.9\left(\mathrm{C}-5-\mathrm{C}_{2} \mathrm{HN}_{3}\right)$, 
$105.1\left(\mathrm{C}-1^{\prime}\right), 103.3(\mathrm{C}-1), 89.2\left(\mathrm{C}_{\mathrm{Cp}}\right), 83.3\left(\mathrm{C}_{\mathrm{Cp}}\right), 80.6(\mathrm{C}-4), 77.0\left(\mathrm{C}-5^{\prime}\right), 76.5(\mathrm{C}-5), 76.2(\mathrm{C}-3), 74.7\left(\mathrm{C}-3^{\prime}\right)$, $74.6(\mathrm{C}-2), 72.5\left(\mathrm{C}-2^{\prime}\right), 70.5\left(\mathrm{C}_{\mathrm{Cp}}\right), 70.4\left(\mathrm{C}_{\mathrm{Cp}}\right), 70.3\left(\mathrm{C}_{\mathrm{Cp}}\right), 70.2\left(\mathrm{C}-4^{\prime}\right), 70.1\left(\mathrm{C}_{\mathrm{Cp}}\right), 63.0\left(\mathrm{CH}_{2} \mathrm{O}\right), 62.5\left(\mathrm{C}-6^{\prime}\right)$, $61.9(\mathrm{C}-6), 60.9\left(\mathrm{CH}_{2} \mathrm{OH}\right), 50.9\left(\mathrm{CH}_{2} \mathrm{~N}_{3}\right) ; \mathrm{m} / z$ (HRESI-TOF): Calc. for $\mathrm{C}_{27} \mathrm{H}_{37} \mathrm{FeN}_{3} \mathrm{O}_{12}$ 651.1727. Found: $652.1215[\mathrm{M}+\mathrm{H}]^{+}, 673.1620[\mathrm{M}+\mathrm{Na}]^{+}$.

2.2.2.

1-(Azidomethyl)-1'-(\{4-[4-O-( $\beta$-D-galactopyranosyl)- $\beta$-D-glucopyranosyloxymethyl]-1H-1,2,3-triazol-1 -yl\}methyl)ferrocene (1)

Compound 5 (650 mg, $0.998 \mathrm{mmol})$ was dissolved in an aqueous solution of $\mathrm{NaN}_{3}(1 \% \mathrm{w} / \mathrm{v})$ acidified with concentrated $\mathrm{HCl}$ until $\mathrm{pH} 1(-0.5 \% \mathrm{HCl})$. The resulting mixture was stirred at room temperature for $2 \mathrm{~h}$. Then, it was neutralized with aqueous $\mathrm{NaOH}$ until $\mathrm{pH} 8$ and lyophilized. The crude was purified by column chromatography on silica gel (EtOAc/MeOH, 2:1) to obtain compound 1 (607 mg, $0.898 \mathrm{mmol}, 90 \%$ ) as a yellow solid. IR $\left(\mathrm{KBr}, \mathrm{cm}^{-1}\right) 3373,2924,2885,2099,1816,1645,1378$, $1328,1259,1238,1225,1156,1116,1058,893,834,790,705,640,507,488 ;{ }^{1} \mathrm{H}$ NMR (300 MHz; $\left.\mathrm{CD}_{3} \mathrm{OD}\right)$ $8.03\left(\mathrm{~s}, 1 \mathrm{H}, \mathrm{H}-5-\mathrm{C}_{2} \mathrm{HN}_{3}\right), 5.38\left(\mathrm{~s}, 2 \mathrm{H}, \mathrm{CH}_{2} \mathrm{~N}_{3}\right), 4.97\left(\mathrm{~d}, 1 \mathrm{H},{ }^{2} \mathrm{~J}=12.4 \mathrm{~Hz}, \mathrm{CHO}\right), 4.79\left(\mathrm{~d}, 1 \mathrm{H},{ }^{2} \mathrm{~J}=12.4 \mathrm{~Hz}\right.$, $\mathrm{CHO}), 4.46\left(\mathrm{~d}, 1 \mathrm{H},{ }^{3} J_{1,2}=6.9 \mathrm{~Hz}, \mathrm{H}-1\right), 4.43-4.37\left(\mathrm{~m}, 3 \mathrm{H}, \mathrm{H}-1^{\prime}, \mathrm{H}_{\mathrm{Cp}}\right), 4.32\left(\mathrm{t}, 2 \mathrm{H},{ }^{3} J=1.6 \mathrm{~Hz}, \mathrm{HCp}\right)$, 4.31-4.25 (m, 4H, $\left.\mathrm{H}_{\mathrm{Cps}}\right), 4.18\left(\mathrm{~s}, 2 \mathrm{H}, \mathrm{CH}_{2} \mathrm{~N}_{3}\right), 4.12-3.72\left(\mathrm{~m}, 5 \mathrm{H}, \mathrm{H}-4^{\prime}, 6,6^{\prime}\right), 3.70-3.46(\mathrm{~m}, 6 \mathrm{H}, \mathrm{H}-2,3$, $\left.3^{\prime}, 4,5,5^{\prime}\right) 3.39-3.28\left(\mathrm{~m}, 1 \mathrm{H}, \mathrm{H}-2, \mathrm{CHD}_{2} \mathrm{OD}\right) ;{ }^{13} \mathrm{C}$ NMR $\left(75 \mathrm{MHz} ; \mathrm{CD}_{3} \mathrm{OD}\right) 145.4\left(\mathrm{C}-4-\mathrm{C}_{2} \mathrm{HN}_{3}\right), 124.9$ $\left(\mathrm{C}-5-\mathrm{C}_{2} \mathrm{HN}_{3}\right), 105.1\left(\mathrm{C}-1^{\prime}\right), 103.2(\mathrm{C}-1), 84.4\left(\mathrm{C}_{\mathrm{Cp}}\right), 83.7\left(\mathrm{C}_{\mathrm{Cp}}\right), 80.6(\mathrm{C}-4), 77.0\left(\mathrm{C}-5^{\prime}\right), 76.5(\mathrm{C}-5), 76.0$ $(\mathrm{C}-3), 74.6\left(\mathrm{C}-2,3^{\prime}\right), 72.4\left(\mathrm{C}-2^{\prime}\right), 70.8\left(\mathrm{C}_{\mathrm{Cp}}\right), 70.7\left(\mathrm{C}_{\mathrm{Cps}}\right), 70.6\left(\mathrm{C}_{\mathrm{Cp}}\right), 70.3\left(\mathrm{C}-4^{\prime}\right), 63.0\left(\mathrm{CH}_{2} \mathrm{O}\right), 62.5\left(\mathrm{C}-6^{\prime}\right)$, 61.7 (C-6), $51.49\left(\mathrm{CH}_{2}-\mathrm{C}_{2} \mathrm{HN}_{3}\right), 50.8\left(\mathrm{CH}_{2} \mathrm{~N}_{3}\right) ; \mathrm{m} / z$ (HRESI-TOF): Calc. for $\mathrm{C}_{27} \mathrm{H}_{36} \mathrm{FeN}_{6} \mathrm{O}_{11} 676.1791$. Found: $652.1377\left[\mathrm{M}-\mathrm{N}_{2}\right]^{+}, 699.1388[\mathrm{M}+\mathrm{Na}]^{+}$.

\subsubsection{General Procedure for the Synthesis of Alkyne-Terminated PAMAM Dendrimers 9-11}

Commercially available $20 \mathrm{wt} \%$ solutions of amine-terminated PAMAM dendrimers 6-8 in methanol were mixed with an equal volume of a solution of $\mathrm{CH}_{2} \mathrm{Cl}_{2}$ containing pent-4-ynoic anhydride ( 3 eq. per amino group). The resulting mixture was stirred at room temperature for 3 days. Diethyl ether was added until a white precipitate appeared. The solid was filtered off, rigorously washed with plenty of diethyl, and used in the next step without further purification.

\section{G0-PAMAM- $\left(\mathrm{COCH}_{2} \mathrm{CH}_{2} \mathrm{C} \equiv \mathrm{CH}\right)_{4}(\mathbf{9})$}

Starting from a $20 \mathrm{wt} \%$ solution of $6(85 \mu \mathrm{L}, 0.028 \mathrm{mmol})$ in methanol, the procedure yielded 9 as a white solid (19 mg, $0.023 \mathrm{mmol}, 81 \%)$. IR $\left(\mathrm{KBr}, \mathrm{cm}^{-1}\right): 3288,3085,2941,2835,1635,1552,1442$, $1374,1298,1272,1240,1126,1099,1016,960,885,731,687,639 ;{ }^{1} \mathrm{H}$ NMR (300 MHz, DMSO- $\left.d_{6}\right): 7.94$ (bs, $8 \mathrm{H}, \mathrm{NH}), 3.07$ (bs, 16H, $\left.\mathrm{CH}_{2} \mathrm{NHCO}\right), 2.76\left(\mathrm{t}, 4 \mathrm{H},{ }^{4} \mathrm{~J}=2.5 \mathrm{~Hz}, \mathrm{HC} \equiv \mathrm{C}\right), 2.63\left(\mathrm{t}, 8 \mathrm{H},{ }^{3} \mathrm{~J}=6.8 \mathrm{~Hz}\right.$, $\mathrm{CH} 2 \mathrm{~N}), 2.42\left(\mathrm{bs}, 4 \mathrm{H}, \mathrm{CH}_{2} \mathrm{~N}\right), 2.38-2.31\left(\mathrm{~m}, 8 \mathrm{H}, \mathrm{CH}_{2} \mathrm{C} \equiv \mathrm{CH}\right), 2.29-2.22\left(\mathrm{~m}, 8 \mathrm{H}, \mathrm{CH}_{2} \mathrm{CO}\right), 2.31\left(\mathrm{t}, 8 \mathrm{H},{ }^{3} \mathrm{~J}\right.$ $\left.=6.8 \mathrm{~Hz}, \mathrm{CH}_{2} \mathrm{CO}\right) ;{ }^{13} \mathrm{C}$ NMR $\left(75 \mathrm{MHz}, \mathrm{DMSO}-d_{6}\right): 171.5(\mathrm{CO}), 170.4(\mathrm{CO}), 83.7(\mathrm{C} \equiv \mathrm{CH}), 71.3(\mathrm{C} \equiv \mathrm{CH})$, $50.9(\mathrm{CH} 2 \mathrm{~N}), 49.6(\mathrm{CH} 2 \mathrm{~N}), 38.4\left(\mathrm{CH}_{2} \mathrm{NHCO}\right), 38.3\left(\mathrm{CH}_{2} \mathrm{NHCO}\right), 34.2\left(\mathrm{CH}_{2} \mathrm{CO}\right), 33.2\left(\mathrm{CH}_{2} \mathrm{CO}\right), 14.2$ $\left(\mathrm{CH}_{2} \mathrm{C} \equiv \mathrm{CH}\right) ; m / z$ (ESI-TOF): Calc. for $\mathrm{C}_{42} \mathrm{H}_{64} \mathrm{~N}_{10} \mathrm{O}_{8}$ 836.5. Found: $841.5[\mathrm{M}+5 \mathrm{H}]^{+}$.

\section{G1-PAMAM- $\left(\mathrm{COCH}_{2} \mathrm{CH}_{2} \mathrm{C} \equiv \mathrm{CH}\right)_{8}(\mathbf{1 0})$}

Starting from a $20 \mathrm{wt} \%$ solution of $7(204 \mu \mathrm{L}, 0.023 \mathrm{mmol})$ in methanol, the procedure yielded 10 as a white solid (37 mg, $0.018 \mathrm{mmol}, 78 \%)$. IR $\left(\mathrm{KBr}, \mathrm{cm}^{-1}\right): 3279,3081,2936,2835,2117,1635,1537$, 1430, 1362, 1234, 1164, 1125, 1037, 644; ${ }^{1} \mathrm{H}$ NMR (300 MHz, DMSO- $\left.d_{6}\right): 8.21-7.65$ (bs, 20H, NH), 3.08 (bs, $40 \mathrm{H}, \mathrm{CH}_{2} \mathrm{NHCO}$ ), 2.74 (bs, $\left.8 \mathrm{H}, \mathrm{HC} \equiv \mathrm{C}\right), 2.64$ (bs, $\left.24 \mathrm{H}, \mathrm{CH}_{2} \mathrm{~N}\right), 2.45-2.14\left(\mathrm{~m}, 68 \mathrm{H}, \mathrm{CH}_{2} \mathrm{~N}, \mathrm{CH}_{2} \mathrm{C} \equiv\right.$ $\left.\mathrm{CH}, \mathrm{CH}_{2} \mathrm{CO}\right) ;{ }^{13} \mathrm{C}$ NMR $\left(75 \mathrm{MHz}\right.$, DMSO-d $\left.d_{6}\right): 172.0(\mathrm{CO}), 170.9(\mathrm{CO}), 84.1(\mathrm{C} \equiv \mathrm{C}), 71.7(\mathrm{C} \equiv \mathrm{CH}), 52.5$ $(\mathrm{CH} 2 \mathrm{~N}), 49.9\left(\mathrm{CH}_{2} \mathrm{~N}\right), 38.8\left(\mathrm{CH}_{2} \mathrm{NHCO}\right), 38.7\left(\mathrm{CH}_{2} \mathrm{NHCO}\right), 37.3\left(\mathrm{CH}_{2} \mathrm{NHCO}\right), 34.9\left(\mathrm{CH}_{2} \mathrm{CO}\right), 34.6$ $\left(\mathrm{CH}_{2} \mathrm{CO}\right), 33.6\left(\mathrm{CH}_{2} \mathrm{CO}\right), 14.6\left(\mathrm{CH}_{2} \mathrm{C} \equiv \mathrm{CH}\right) ; \mathrm{m} / z$ (ESI-TOF): Calc. for $\mathrm{C}_{102} \mathrm{H}_{160} \mathrm{~N}_{26} \mathrm{O}_{20}$ 2070.2. Found: $2071.2[\mathrm{M}+\mathrm{H}]^{+}$. 


\section{G2-PAMAM- $\left(\mathrm{COCH}_{2} \mathrm{CH}_{2} \mathrm{C} \equiv \mathrm{CH}\right)_{16}(\mathbf{1 1})$}

Starting from a $20 \mathrm{wt} \%$ solution of $8(221 \mu \mathrm{L}, 0.012 \mathrm{mmol})$ in methanol, the procedure yielded 11 as a white solid (43 mg, $0.009 \mathrm{mmol}, 79 \%)$. IR $\left(\mathrm{KBr}, \mathrm{cm}^{-1}\right)$ : 3286, 3084, 2940, 2835, 1633, 1540, 1432, 1368, $1238,1167,1125,1023,638 ;{ }^{1} \mathrm{H}$ NMR $\left(300 \mathrm{MHz}, \mathrm{DMSO}-d_{6}\right): 8.39-7.58(\mathrm{bs}, 44 \mathrm{H}, \mathrm{NH}), 3.08$ (bs, $88 \mathrm{H}$, $\mathrm{CH}_{2} \mathrm{NHCO}$ ), 2.80-2.59 (bs, 72H, HC $\left.\equiv \mathrm{C}, \mathrm{CH} 2 \mathrm{~N}\right), 2.46-2.04\left(\mathrm{~m}, 148 \mathrm{H}, \mathrm{CH}_{2} \mathrm{~N}, \mathrm{CH}_{2} \equiv \mathrm{CH}_{2} \mathrm{CH}_{2} \mathrm{CO}\right) ;{ }^{13} \mathrm{C}$ NMR (75 MHz, DMSO- $\left.d_{6}\right)$ : $172.0(\mathrm{CO}), 171.7(\mathrm{CO}), 170.9(\mathrm{CO}), 84.1(\mathrm{C} \equiv \mathrm{CH}), 71.7(\mathrm{C} \equiv \mathrm{CH}), 52.5\left(\mathrm{CH}_{2} \mathrm{~N}\right)$, $49.9\left(\mathrm{CH}_{2} \mathrm{~N}\right), 38.8\left(\mathrm{CH}_{2} \mathrm{NHCO}\right), 38.7\left(\mathrm{CH}_{2} \mathrm{NHCO}\right), 37.3\left(\mathrm{CH}_{2} \mathrm{NHCO}\right), 34.6\left(\mathrm{CH}_{2} \mathrm{CO}\right), 33.6\left(\mathrm{CH}_{2} \mathrm{CO}\right)$, $14.6\left(\mathrm{CH}_{2} \mathrm{C} \equiv \mathrm{CH}\right) ; \mathrm{m} / z$ (ESI-TOF): Calc. for $\mathrm{C}_{222} \mathrm{H}_{353} \mathrm{~N}_{58} \mathrm{O}_{44}$ 4536.7157. Found: $4538.7[\mathrm{M}+4 \mathrm{H}]^{+}$.

\subsubsection{General Procedure for the Synthesis of Electroactive Lactosylated PAMAM Dendrimers 12-14}

The alkyne-terminated PAMAM dendrimers (9-11) and $\mathbf{1}$ (1.5 eq. per alkyne group) were dissolved in $\mathrm{THF} / \mathrm{H}_{2} \mathrm{O}$ 1:1 (0.04 mL per mg of 5) under $\mathrm{N}_{2}$ atmosphere. Then, $\mathrm{CuSO}_{4}$ (0.2 eq. per alkyne group) and sodium ascorbate ( 0.4 eq. per alkyne group) were added to the solution in this order. The resulting mixture was stirred at room temperature for $20 \mathrm{~h}$. After that, THF was evaporated under vacuum without heating over $40^{\circ} \mathrm{C}$. The resulting solution was diluted until $-10 \mathrm{~mL}$ with $5 \%$ aqueous $\mathrm{NH}_{3}$ and the product was isolated by repetitive centrifugal filtration ( $3 \mathrm{kDa}$ cut-off, $10 \times$ dilution) with 5 consecutive concentration and re-dissolution steps. The final solution was lyophilized to obtain the corresponding electroactive lactosylated PAMAM dendrimer as a yellow solid.

\section{G0-PAMAM- $\left(\mathrm{COCH}_{2} \mathrm{CH}_{2} \mathrm{TACH}_{2} \mathrm{FCCH}_{2} \mathrm{TACH}_{2} \mathrm{Lac}\right)_{4}$ (12)}

Starting from 9 (21 mg, $0.025 \mathrm{mmol})$ in methanol, the procedure yielded 12 as a yellow solid (64 mg, $0.018 \mathrm{mmol}, 72 \%)$. IR (KBr, cm ${ }^{-1}$ ): 3412, 2930, 1646, 1553, 1435, 1383, 1338, 1237, 1126, 1058, 815, 712, 623, 508, 493; ${ }^{1} \mathrm{H}$ NMR (300 MHz, DMSO-d $\left.d_{6}\right): 8.06$ (s, 4H, H5- $\left.{ }_{2} \mathrm{HN}_{3}\right), 8.00-7.85(\mathrm{~m}, 8 \mathrm{H}$, $\mathrm{NH}), 7.76\left(\mathrm{~s}, 4 \mathrm{H}, \mathrm{H}^{\prime}-\mathrm{C}_{2} \mathrm{HN}_{3}\right), 5.32\left(\mathrm{~s}, 8 \mathrm{H}, \mathrm{CH}_{2} \mathrm{~N}_{3}\right), 5.27\left(\mathrm{~s}, 8 \mathrm{H}, \mathrm{CH}_{2} \mathrm{~N}_{3}\right), 5.20-5.05(\mathrm{~m}, 8 \mathrm{H}, \mathrm{OHs}), 4.83$ $\left(\mathrm{d}, 4 \mathrm{H},{ }^{2} \mathrm{~J}=12.4 \mathrm{~Hz}, \mathrm{CHO}\right), 4.82-4.76(\mathrm{~m}, 8 \mathrm{H}, \mathrm{OHs}), 4.73-4.60(\mathrm{~m}, 12 \mathrm{H}, \mathrm{OHs}), 4.61\left(\mathrm{~d}, 4 \mathrm{H},{ }^{2} \mathrm{~J}=12.4\right.$ $\mathrm{Hz}, \mathrm{CHO}), 4.39-4.29\left(\mathrm{~m}, 20 \mathrm{H}, \mathrm{H}_{\mathrm{Cp}}, \mathrm{H}-1^{\prime}\right), 4.23-4.16$ (m, 20H, $\left.\mathrm{H}_{\mathrm{Cp}}, \mathrm{H}-1\right), 3.80$ (dd, $4 \mathrm{H},{ }^{2} J_{6^{\prime}, 6}=11.2$ $\left.\mathrm{Hz},{ }^{3} J_{6^{\prime}, 5}=11.2 \mathrm{~Hz}, \mathrm{H}-6^{\prime}\right), 3.65-3.40\left(\mathrm{~m}, 16 \mathrm{H}, \mathrm{H}-4^{\prime}, 5,5^{\prime}, 6\right), 3.39-3.18$ (m, H-2, 2', 3, 3' , 4, HDO), 3.05 (bs, $\left.16 \mathrm{H}, \mathrm{CH}_{2} \mathrm{NHCO}\right), 2.81\left(\mathrm{t}, 8 \mathrm{H},{ }^{3} \mathrm{~J}=7.6 \mathrm{~Hz}, \mathrm{CH}_{2}-\mathrm{C}_{2} \mathrm{HN}_{3}\right), 2.63\left(\mathrm{bs}, 8 \mathrm{H}, \mathrm{CH}_{2} \mathrm{~N}\right), 2.45-2.33(\mathrm{~m}, 12 \mathrm{H}$, $\mathrm{CH}_{2} \mathrm{~N}, \mathrm{CH}_{2} \mathrm{CO}$ ), 2.19 (bs, 8H, $\left.\mathrm{CH}_{2} \mathrm{CO}\right) ;{ }^{13} \mathrm{C}$ NMR (75 MHz, DMSO- $d_{6}$ ): 171.5 (CO), 171.3 (CO), 146.0 $\left(\mathrm{C} 4-\mathrm{C}_{2} \mathrm{HN}_{3}\right), 143.7\left(\mathrm{C} 4-\mathrm{C}_{2} \mathrm{HN}_{3}\right), 123.7\left(\mathrm{C}^{2}-\mathrm{C}_{2} \mathrm{HN}_{3}\right), 121.4\left(\mathrm{C} 5-\mathrm{C}_{2} \mathrm{HN}_{3}\right), 103.8\left(\mathrm{C}-1^{\prime}\right), 101.8(\mathrm{C}-1), 83.2$ $\left(\mathrm{C}_{\mathrm{Cp}}\right), 82.9\left(\mathrm{C}_{\mathrm{Cp}}\right), 80.7(\mathrm{C}-4), 75.5\left(\mathrm{C}-5^{\prime}\right), 74.9(\mathrm{C}-3,5), 73.2\left(\mathrm{C}-2^{\prime}\right), 73.0(\mathrm{C}-2), 70.5\left(\mathrm{C}-3^{\prime}\right), 69.6\left(\mathrm{C}_{\mathrm{Cp}}\right), 69.5$ $\left(\mathrm{C}_{\mathrm{Cp}}\right), 69.3\left(\mathrm{C}_{\mathrm{Cp}}\right), 69.2\left(\mathrm{C}_{\mathrm{Cp}}\right), 68.1\left(\mathrm{C}-4^{\prime}\right), 61.7\left(\mathrm{CH}_{2} \mathrm{O}\right), 60.5(\mathrm{C}-6), 60.4\left(\mathrm{C}-6^{\prime}\right), 50.8\left(\mathrm{CH}_{2} \mathrm{~N}\right), 49.6\left(\mathrm{CH}_{2} \mathrm{~N}\right)$, $48.6\left(\mathrm{CH}_{2} \mathrm{~N}_{3}\right), 48.5\left(\mathrm{CH}_{2} \mathrm{~N}_{3}\right), 38.3(\mathrm{CH} 2 \mathrm{NHCO}), 34.9\left(\mathrm{CH}_{2} \mathrm{CO}\right), 33.2\left(\mathrm{CH}_{2} \mathrm{CO}\right), 21.2\left(\mathrm{CH}_{2}-\mathrm{C}_{2} \mathrm{HN}_{3}\right) ; \mathrm{m} / \mathrm{z}$ (HRESI-TOF): Calc. for $\mathrm{C}_{150} \mathrm{H}_{208} \mathrm{Fe}_{4} \mathrm{~N}_{34} \mathrm{O}_{52}$ 3542.2108. Found: $1772.1643[\mathrm{M}+2 \mathrm{H}]^{2+}, 1181.3887[\mathrm{M}+$ $3 \mathrm{H}]^{3+}, 886.2606[\mathrm{M}+4 \mathrm{H}]^{+}$. Number of expected lactose units per dendrimer: 4 . Found by sulfuric acid-phenol assays: $4.10 \pm 0.21$ (see the Supplementary Materials).

\section{G1-PAMAM- $\left(\mathrm{COCH}_{2} \mathrm{CH}_{2} \mathrm{TACH}_{2} \mathrm{FCCH}_{2} \mathrm{TACH}_{2} \mathrm{Lac}\right)_{8}$ (13)}

Starting from $10(60 \mathrm{mg}, 0.029 \mathrm{mmol})$ in methanol, the procedure yielded 13 as a yellow solid (155 mg, $0.021 \mathrm{mmol}, 73 \%)$. IR $\left(\mathrm{KBr}, \mathrm{cm}^{-1}\right)$ : 3363, 2956, 2922, 2852, 1731, 1653, 1553, 1461, 1435, $1378,1339,1237,1129,1057,973,816,684,642,510,489 ;{ }^{1} \mathrm{H}$ NMR $\left(300 \mathrm{MHz}\right.$, DMSO- $\left.d_{6}\right): 8.06$ (s, 8H, $\left.\mathrm{H} 5-\mathrm{C}_{2} \mathrm{HN}_{3}\right), 8.00-7.81(\mathrm{~m}, 20 \mathrm{H}, \mathrm{NH}), 7.76\left(\mathrm{~s}, 8 \mathrm{H}, \mathrm{H}^{\prime}-\mathrm{C}_{2} \mathrm{HN}_{3}\right), 5.31\left(\mathrm{~s}, 16 \mathrm{H}, \mathrm{CH}_{2} \mathrm{~N}_{3}\right), 5.27(\mathrm{~s}, 16 \mathrm{H}$, $\left.\mathrm{CH}_{2} \mathrm{~N}_{3}\right), 5.19$ (bs, 8H, OHs), 5.11 (bs, $\left.8 \mathrm{H}, \mathrm{OHs}\right), 4.89-4.76(\mathrm{~m}, 16 \mathrm{H}, \mathrm{CHO}, \mathrm{OH}), 4.74-4.48(\mathrm{~m}, 40 \mathrm{H}, \mathrm{CHO}$, $\mathrm{OHs}), 4.39-4.27\left(\mathrm{~m}, 40 \mathrm{H}, \mathrm{H}_{\mathrm{Cp}}, \mathrm{H}-1^{\prime}\right), 4.26-4.15\left(\mathrm{~m}, 40 \mathrm{H}, \mathrm{H}_{\mathrm{Cp}}, \mathrm{H}-1\right), 3.84-3.74\left(\mathrm{~m}, 8 \mathrm{H}, \mathrm{H}-6^{\prime}\right), 3.65-3.34$ (m, 64H, H-4', 5, 5' $\left., 6,2,2^{\prime}, 3,3^{\prime}, 4\right), 3.06$ (bs, 40H, CH $\left.\mathrm{CH}_{2} \mathrm{NHCO}\right), 2.81\left(\mathrm{t}, 16 \mathrm{H},{ }^{3} \mathrm{~J}=7.0 \mathrm{~Hz}, \mathrm{CH}_{2}-\mathrm{C}_{2} \mathrm{HN}_{3}\right)$, 2.64 (bs, $\left.24 \mathrm{H}, \mathrm{CH}_{2} \mathrm{~N}\right), 2.45-2.33\left(\mathrm{~m}, 28 \mathrm{H}, \mathrm{CH}_{2} \mathrm{~N}, \mathrm{CH}_{2} \mathrm{CO}\right), 2.18\left(\mathrm{bs}, 24 \mathrm{H}, \mathrm{CH}_{2} \mathrm{CO}\right) ;{ }^{13} \mathrm{C} \mathrm{NMR}(75 \mathrm{MHz}$, DMSO- $\left.d_{6}\right)$ : $171.6(\mathrm{CO}), 171.4(\mathrm{CO}), 171.3(\mathrm{CO}), 146.0\left(\mathrm{C} 4-\mathrm{C}_{2} \mathrm{HN}_{3}\right), 143.7\left(\mathrm{C}_{-}-\mathrm{C}_{2} \mathrm{HN}_{3}\right), 123.7\left(\mathrm{C} 5-\mathrm{C}_{2} \mathrm{HN}_{3}\right)$, 121.4 (C5- $\left.\mathrm{C}_{2} \mathrm{HN}_{3}\right), 103.9\left(\mathrm{C}-1^{\prime}\right), 101.9(\mathrm{C}-1), 83.1\left(\mathrm{C}_{\mathrm{Cp}}\right), 82.8\left(\mathrm{C}_{\mathrm{Cp}}\right), 80.7(\mathrm{C}-4), 75.5\left(\mathrm{C}-5^{\prime}\right), 74.9(\mathrm{C}-3$, 5), $73.2\left(\mathrm{C}-2^{\prime}\right), 73.0(\mathrm{C}-2), 70.5\left(\mathrm{C}-3^{\prime}\right), 69.6\left(\mathrm{C}_{\mathrm{Cps}}\right), 69.3\left(\mathrm{C}_{\mathrm{Cp}}\right), 69.2\left(\mathrm{C}_{\mathrm{Cp}}\right), 68.1\left(\mathrm{C}-4^{\prime}\right), 61.7\left(\mathrm{CH}_{2} \mathrm{O}\right)$, 
$60.5(\mathrm{C}-6), 60.4\left(\mathrm{C}-6^{\prime}\right), 52.2\left(\mathrm{CH}_{2} \mathrm{~N}\right), 49.5\left(\mathrm{CH}_{2} \mathrm{~N}\right), 48.6\left(\mathrm{CH}_{2} \mathrm{~N}_{3}\right), 48.5\left(\mathrm{CH}_{2} \mathrm{~N}_{3}\right), 38.4\left(\mathrm{CH}_{2} \mathrm{NHCO}\right), 38.3$ ( $\left.\mathrm{CH}_{2} \mathrm{NHCO}\right), 36.9\left(\mathrm{CH}_{2} \mathrm{NHCO}\right), 34.9\left(\mathrm{CH}_{2} \mathrm{CO}\right), 33.2\left(\mathrm{CH}_{2} \mathrm{CO}\right), 21.2\left(\mathrm{CH}_{2}-\mathrm{C}_{2} \mathrm{HN}_{3}\right) ; m / z(\mathrm{MALDI}-\mathrm{TOF})$ : Calc. for $\mathrm{C}_{318} \mathrm{H}_{448} \mathrm{Fe}_{8} \mathrm{~N}_{74} \mathrm{O}_{108} 7481.7$ (EM); Found: 7481.3 [M] ${ }^{+}$. Number of expected lactose units per dendrimer: 8 . Found by sulfuric acid-phenol assays: $7.97 \pm 0.23$ (see Supplementary Materials).

\section{G2-PAMAM- $\left(\mathrm{COCH}_{2} \mathrm{CH}_{2} \mathrm{TACH}_{2} \mathrm{FcCH}_{2} \mathrm{TACH}_{2} \mathrm{Lac}\right)_{16}$ (14)}

Starting from 11 (40 mg, $0.009 \mathrm{mmol}$ ) in methanol, the procedure yielded 14 as a yellow solid (101 mg, $0.007 \mathrm{mmol}, 73 \%)$. IR (KBr, cm $\left.{ }^{-1}\right): 3415,2931,1643,1552,1436,1373,1337,1327,1128,1056$, 813, 716, 682, 591, 493; ${ }^{1} \mathrm{H}$ NMR (300 MHz, DMSO- $\left.d_{6}\right): 8.06$ (s, $\left.16 \mathrm{H}, \mathrm{H} 5-\mathrm{C}_{2} \mathrm{HN}_{3}\right), 8.00-7.77(\mathrm{~m}, 44 \mathrm{H}$, $\mathrm{NH}), 7.76\left(\mathrm{~s}, 16 \mathrm{H}, \mathrm{H}^{\prime}-\mathrm{C}_{2} \mathrm{HN}_{3}\right), 5.31\left(\mathrm{~s}, 32 \mathrm{H}, \mathrm{CH}_{2} \mathrm{~N}_{3}\right), 5.27\left(\mathrm{~s}, 32 \mathrm{H}, \mathrm{CH}_{2} \mathrm{~N}_{3}\right), 5.20$ (bs, $\left.16 \mathrm{H}, \mathrm{OHs}\right), 5.11$ (bs, $16 \mathrm{H}, \mathrm{OHs}), 4.89-4.75(\mathrm{~m}, 32 \mathrm{H}, \mathrm{CHO}, \mathrm{OH}), 4.74-4.47$ (m, 80H, CHO, OHs), $4.39-4.28(\mathrm{~m}, 80 \mathrm{H}$, $\left.\mathrm{H}_{\mathrm{Cp}}, \mathrm{H}-1^{\prime}\right), 4.26-4.10\left(\mathrm{~m}, 80 \mathrm{H}, \mathrm{H}_{\mathrm{Cp}}, \mathrm{H}-1\right), 3.87-3.74$ (m, 16H, H-6'), 3.70-3.41 (m, 64H, H-4' ,5, 5',6), 3.40-3.21 (m, H-2, 2' , 3, 3' , 4, HDO), 3.06 (bs, $\left.84 \mathrm{H}, \mathrm{CH}_{2} \mathrm{NHCO}\right), 2.81\left(\mathrm{t}, 32 \mathrm{H},{ }^{3} \mathrm{~J}=7.3 \mathrm{~Hz}, \mathrm{CH}_{2}-\mathrm{C}_{2} \mathrm{HN}_{3}\right.$ ), 2.65 (bs, 56H, CH2N), 2.48-2.31 (m, 60H, $\left.\mathrm{CH}_{2} \mathrm{~N}, \mathrm{CH}_{2} \mathrm{CO}\right), 2.18$ (bs, 56, $\left.\mathrm{CH}_{2} \mathrm{CO}\right) ;{ }^{13} \mathrm{C} \mathrm{NMR}(75 \mathrm{MHz}$, DMSO- $\left.d_{6}\right)$ : $171.6(\mathrm{CO}), 171.4(\mathrm{CO}), 171.3(\mathrm{CO}), 146.0\left(\mathrm{C} 4-\mathrm{C}_{2} \mathrm{HN}_{3}\right), 143.7\left(\mathrm{C} 4-\mathrm{C}_{2} \mathrm{HN}_{3}\right), 123.7\left(\mathrm{C} 5-\mathrm{C}_{2} \mathrm{HN}_{3}\right)$, $121.4\left(\mathrm{C}^{-} \mathrm{C}_{2} \mathrm{HN}_{3}\right), 103.9\left(\mathrm{C}-1^{\prime}\right), 101.9(\mathrm{C}-1), 83.1\left(\mathrm{C}_{\mathrm{Cp}}\right), 82.9\left(\mathrm{C}_{\mathrm{Cp}}\right), 80.7(\mathrm{C}-4), 75.5\left(\mathrm{C}-5^{\prime}\right), 74.9(\mathrm{C}-3,5)$, $73.2\left(\mathrm{C}-2^{\prime}\right), 73.0(\mathrm{C}-2), 70.5\left(\mathrm{C}-3^{\prime}\right), 69.7\left(\mathrm{C}_{\mathrm{Cp}}\right), 69.6\left(\mathrm{C}_{\mathrm{Cp}}\right), 69.4\left(\mathrm{C}_{\mathrm{Cp}}\right), 69.3\left(\mathrm{C}_{\mathrm{Cp}}\right), 68.2\left(\mathrm{C}-4^{\prime}\right), 61.7\left(\mathrm{CH}_{2} \mathrm{O}\right)$, $60.5(\mathrm{C}-6), 60.4\left(\mathrm{C}-6^{\prime}\right), 52.1\left(\mathrm{CH}_{2} \mathrm{~N}\right), 49.5\left(\mathrm{CH}_{2} \mathrm{~N}\right), 48.7\left(\mathrm{CH}_{2} \mathrm{~N}_{3}\right), 48.5\left(\mathrm{CH}_{2} \mathrm{~N}_{3}\right), 38.4(\mathrm{CH} 2 \mathrm{NHCO}), 38.3$ $\left(\mathrm{CH}_{2} \mathrm{NHCO}\right), 36.9\left(\mathrm{CH}_{2} \mathrm{NHCO}\right), 34.9\left(\mathrm{CH}_{2} \mathrm{CO}\right), 33.2\left(\mathrm{CH}_{2} \mathrm{CO}\right), 21.2\left(\mathrm{CH}_{2}-\mathrm{C}_{2} \mathrm{HN}_{3}\right) ; \mathrm{m} / z$ (ESI-TOF): Calc. for $\mathrm{C}_{654} \mathrm{H}_{928} \mathrm{Fe}_{16} \mathrm{~N}_{154} \mathrm{O}_{220} 15361$ Found: $15361[\mathrm{M}]^{+}$. Number of expected lactose units per dendrimer: 16. Found by sulfuric acid-phenol assays: $16.35 \pm 1.11$ (see Supplementary Materials).

2.2.5. Bis [2 3-(3-\{1-[1'-(\{4-[4-O-( $\beta$-D-galactopyranosyl)- $\beta$-D-glucopyranosyloxymethyl]-1H-1,2,3triazol-1-yl\}methyl)ferrocenylmethyl]-1H-1,2,3-triazol-4-yl\}propanoylamino)-12,15,18,21-tetr aoxatricosanyl] disulfide (17)

$\mathrm{CuSO}_{4}(7 \mathrm{mg}, 0.032 \mathrm{mmol})$ and sodium ascorbate $(10 \mathrm{mg}, 0.063 \mathrm{mmol})$ were added to a stirred solution of compounds 16 (70 mg, $0.076 \mathrm{mmol})$ and $1(113 \mathrm{mg}, 0.167 \mathrm{mmol})$ in THF/ $\mathrm{H}_{2} \mathrm{O}(6 \mathrm{~mL}, 1: 1)$ under $\mathrm{N}_{2}$. The resulting mixture was stirred at room temperature overnight and then the solvent was evaporated to dryness at reduced pressure. The crude was purified by column chromatography on silica gel $\left(\mathrm{CH}_{3} \mathrm{CN} / \mathrm{H}_{2} \mathrm{O} / \mathrm{NH}_{3(\mathrm{aq} \cdot)}\right.$ 30\%, 8:2:1 $\rightarrow$ 10:4) to obtain compound $17(164 \mathrm{mg}, 0.072 \mathrm{mmol}, 95 \%)$ as a yellow solid. IR $\left(\mathrm{KBr}, \mathrm{cm}^{-1}\right)$ : 3390, 2931, 1635, 1237, 1167, 1126, 1097, 1079, 1061, 1025, 840, 815,

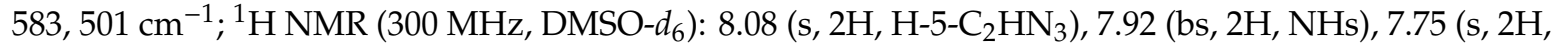
$\left.\mathrm{H}-5-\mathrm{C}_{2} \mathrm{HN}_{3}\right), 5.31\left(\mathrm{~s}, 4 \mathrm{H}, \mathrm{CH}_{2}-\mathrm{C}_{2} \mathrm{HN}_{3}\right), 5.26\left(\mathrm{~s}, 4 \mathrm{H}, \mathrm{CH}_{2}-\mathrm{C}_{2} \mathrm{HN}_{3}\right), 4.82\left(\mathrm{~d},{ }^{2} \mathrm{~J}=12.0 \mathrm{~Hz}, 2 \mathrm{H}, \mathrm{CHO}\right), 4.60$ $\left(\mathrm{d},{ }^{2} \mathrm{~J}=12.0 \mathrm{~Hz}, 2 \mathrm{H}, \mathrm{CHO}\right), 4.40-4.27\left(\mathrm{~m}, 6 \mathrm{H}, \mathrm{H}_{\mathrm{Cp}}, \mathrm{H}-1\right), 4.24-4.14\left(\mathrm{~m}, 6 \mathrm{H}, \mathrm{H}_{\mathrm{Cp}}, \mathrm{H}-\mathrm{1}^{\prime}\right), 3.86-3.74(\mathrm{~m}$, 6H, H-2, 6), 3.67-3.13 (m, H-2', 3, 3' $\left., 4,4^{\prime}, 5,5^{\prime}, 6^{\prime}, \mathrm{CH}_{2} \mathrm{OEG}, \mathrm{CH}_{2} \mathrm{NHCO}, \mathrm{OCH}_{2}\left(\mathrm{CH}_{2}\right)_{10}, \mathrm{HDO}\right), 3.01$ $\left(\mathrm{t},{ }^{3} \mathrm{~J}=7.5 \mathrm{~Hz}, 4 \mathrm{H}, \mathrm{CH}_{2} \mathrm{CONH}\right), 2.80\left(\mathrm{t},{ }^{3} \mathrm{~J}=7.0 \mathrm{~Hz}, 4 \mathrm{H}, \mathrm{CH}_{2}-\mathrm{C}_{2} \mathrm{HN}_{3}\right), 2.67\left(\mathrm{t},{ }^{3} \mathrm{~J}=7.1 \mathrm{~Hz}, 4 \mathrm{H}, \mathrm{CH}_{2} \mathrm{~S}\right)$, 1.67-1.53 (m, 4H, $\left.\left(\mathrm{CH}_{2}\right)_{9}\right), 1.52-1.39\left(\mathrm{~m}, 4 \mathrm{H},\left(\mathrm{CH}_{2}\right)_{9}\right), 1.38-1.12\left(\mathrm{~m}, 28 \mathrm{H},\left(\mathrm{CH}_{2}\right)_{9}\right) ;{ }^{13} \mathrm{C} \mathrm{NMR}(75 \mathrm{MHz}$, DMSO- $\left.d_{6}\right)$ : $171.6(\mathrm{CO}), 146.0\left(\mathrm{C}_{4}-\mathrm{C}_{2} \mathrm{HN}_{3}\right), 143.7\left(\mathrm{C} 4-\mathrm{C}_{2} \mathrm{HN}_{3}\right), 123.7\left(\mathrm{C}^{-}-\mathrm{C}_{2} \mathrm{HN}_{3}\right), 121.4\left(\mathrm{C} 5-\mathrm{C}_{2} \mathrm{HN}_{3}\right)$, 103.8 (C-1'), 101.8 (C-1), 83.2 ( $\left.\mathrm{C}_{\mathrm{Cp}}\right), 82.9\left(\mathrm{C}_{\mathrm{Cp}}\right), 80.7(\mathrm{C}-4), 75.5\left(\mathrm{C}-5^{\prime}\right), 74.9(\mathrm{C}-3,5), 73.2\left(\mathrm{C}-2^{\prime}\right), 73.1(\mathrm{C}-2)$, $70.5\left(\mathrm{C}-3^{\prime}\right), 70.3\left(\mathrm{CH}_{2} \mathrm{OEG}\right), 69.8\left(\mathrm{CH}_{2} \mathrm{OEG}\right), 69.7\left(\mathrm{CH}_{2} \mathrm{OEG}\right), 69.6\left(\mathrm{C}_{\mathrm{Cp}}\right), 69.5\left(\mathrm{C}_{\mathrm{Cp}}\right), 69.4\left(\mathrm{CH}_{2} \mathrm{OEG}\right)$, $69.3\left(\mathrm{C}_{\mathrm{Cp}}\right), 69.2\left(\mathrm{C}_{\mathrm{Cp}}\right), 69.1\left(\mathrm{CH}_{2} \mathrm{OEG}\right), 68.1\left(\mathrm{C}-4^{\prime}\right), 61.7\left(\mathrm{CH}_{2} \mathrm{O}\right), 60.5(\mathrm{C}-6), 60.4\left(\mathrm{C}-6^{\prime}\right), 48.6\left(\mathrm{CH}_{2} \mathrm{~N}_{3}\right)$, $48.4\left(\mathrm{CH}_{2} \mathrm{~N}_{3}\right), 38.5\left(\mathrm{CH}_{2} \mathrm{NHCO}\right), 37.9\left(\mathrm{CH}_{2} \mathrm{~S}\right), 34.7\left(\mathrm{CH}_{2} \mathrm{CONH}\right), 29.2,29.0,28.9,28.5,25.6\left[\left(\mathrm{CH}_{2}\right)_{9}\right], 21.2$ $\left(\mathrm{CH}_{2}-\mathrm{C}_{2} \mathrm{HN}_{3}\right) ; m / z$ (ESI-TOF): Calc. for $\mathrm{C}_{102} \mathrm{H}_{160} \mathrm{Fe}_{2} \mathrm{~N}_{14} \mathrm{O}_{32} \mathrm{~S}_{2} 2269.9497$ Found: $2270.5857[\mathrm{M}+\mathrm{H}]^{+}$.

\subsubsection{Synthesis of Citrate-Stabilized AuNPs}

Citrate-stabilized AuNPs were prepared according to a modification [46] of the Turkevich protocol [47]. Briefly, a degassed solution of sodium citrate $(228 \mathrm{mg}, 0.775 \mathrm{mmol})$ in Milli-Q water $(20 \mathrm{~mL})$ at $55{ }^{\circ} \mathrm{C}$ was added to a refluxing degassed solution of $\mathrm{HAuCl}_{4} \cdot 3 \mathrm{H}_{2} \mathrm{O}(79 \mathrm{mg}, 0.201 \mathrm{mmol})$ in Milli-Q water $(200 \mathrm{~mL})$ under vigorous stirring. The mixture was refluxed for $30 \mathrm{~min}$ and cooled to room temperature before being filtered through a Sartorius Minisart $0.2 \mu \mathrm{m}$ filter to provide citrate-stabilized 
AuNPs of $12.1 \pm 1.0 \mathrm{~nm}$ of average diameter in a concentration of $16 \mathrm{nM}$, as determined by UV-vis spectroscopy [48] and TEM. The citrate-stabilized AuNPs solution was washed with $1 \mathrm{mM} \mathrm{NaOH}[36,49]$ by repetitive centrifugal filtration ( $10 \mathrm{kDa}$ cut-off, $10 \times$ dilution) with three consecutive concentration and re-dissolution steps to obtain $16 \mathrm{nM}$ AuNPs solution in $1 \mathrm{mM} \mathrm{NaOH}$ with an average size of $12.1 \pm 1.2 \mathrm{~nm}$ as determined by TEM.

\subsubsection{Preparation of AuNP@Fc-Lac}

A $2.5 \mathrm{mM}$ solution of disulfide 17 in methanol $(2 \mathrm{~mL})$ was added to a stirred solution of $16 \mathrm{nM}$ citrate-stabilized AuNPs in $1 \mathrm{mM} \mathrm{NaOH}(10 \mathrm{~mL})$ and the resulting solution was stirred for $24 \mathrm{~h}$. After that, THF $(10 \mathrm{~mL})$ was added and the resulting suspension was precipitated by centrifugation at $4000 \mathrm{rpm}$ for $20 \mathrm{~min}$. The supernatant was removed and the functionalized AuNPs were re-dissolved in $\mathrm{H}_{2} \mathrm{O}(10 \mathrm{~mL})$. THF $(10 \mathrm{~mL})$ was added and the resulting suspension was precipitated again by centrifugation at $4000 \mathrm{rpm}$ for $20 \mathrm{~min}$. The process was repeated 3 times. After that, the functionalized AuNPs were dissolved in $10 \mathrm{~mm}$ phosphate buffer ( $\mathrm{pH}$ 7.2) and dialyzed by centrifugal filtration (10 x dilution, Millipore Amicon Centriplus $10 \mathrm{kDa}$ ). The process was repeated 4 times to obtain a final solution of $20 \mathrm{~nm}$ AuNP@Fc-Lac (8 mL) in $10 \mathrm{~mm}$ phosphate buffer (pH 7.2). Average diameter of the gold core $=12.2 \pm 1.1 \mathrm{~nm}$ (from TEM analysis). Number of ligands/AuNP $=1659 \pm 94$ (from elemental gold-to-iron ratio as determined by ICP-OES). Concentrations were determined by transmission electron microscopy (TEM) and UV-visible spectroscopy [48].

\subsection{UV-Visible Experiments}

UV-visible measurements were performed using Jasco V-530 spectrophotometer at room temperature using a $1 \mathrm{~cm}$ path length. For protein assays, aqueous dispersions of AuNP@Fc-Lac (2 $\mathrm{nM}$ ) in phosphate buffer $10 \mathrm{mM}$ at pH 7.2 with $20 \mathrm{mM} \mathrm{NaCl}$ were treated with a final concentration of $10.0 \mu \mathrm{M}$ of Gal-3 or bovine serum albumin.

\subsection{Voltammetric Experiments}

Electrochemical measurements were carried out in sonicated, nitrogen-purged aqueous (Milli-Q $18.2 \mathrm{M} \Omega \mathrm{cm}$ ) solution with a micro-Autolab type III connected to a personal computer running Eco Chimie B. V. GPES 4.9 software. The electrodes were carefully cleaned before each experiment. The glassy carbon disk working electrode ( $2 \mathrm{~mm}$, effective area $0.038 \pm 0.006 \mathrm{~cm}^{2}$ ) was immersed in a $0.1 \mathrm{M} \mathrm{HNO}_{3}$ solution for $5 \mathrm{~min}$ and polished with a basic $\mathrm{Al}_{2} \mathrm{O}_{3}$ water slurry. The platinum sheet counter electrode $\left(6 \times 4 \mathrm{~mm}\right.$, effective area $\left.0.410 \pm 0.003 \mathrm{~cm}^{2}\right)$ was immersed in a $50 \% v / v \mathrm{H}_{2} \mathrm{SO}_{4}$ solution for $5 \mathrm{~min}$. Both electrodes were then sonicated in a 1:1:1 $\mathrm{H}_{2} \mathrm{O} / \mathrm{MeOH} / \mathrm{CH}_{3} \mathrm{CN}$ mixture for $5 \mathrm{~min}$ prior to use. The effective area of the electrodes was determined as previously reported [39]. An $\mathrm{Ag} / \mathrm{AgCl}$ (3 M KCl) electrode was used as a reference. Solutions of conjugate 18 (Figure 1) and dendrimers 12-14 (50 $\mu \mathrm{M}$ for $18,25 \mu \mathrm{M}$ for $12,12.5 \mu \mathrm{M}$ for 13 and $6.25 \mu \mathrm{M}$ for 14) and increasing amounts of Gal-3 varying from 0 to $45 \mu \mathrm{M}$ were prepared in $10 \mathrm{mM}$ phosphate buffer at $\mathrm{pH} 7.2$ with $20 \mathrm{mM} \mathrm{NaCl}$ and incubated for $10 \mathrm{~min}$ at room temperature. Before each measurement with AuNP@Fc-Lac, the glassy carbon electrode was modified by incubation in solutions of AuNP@Fc-Lac $(20 \mathrm{nM})$ in $10 \mathrm{mM}$ phosphate buffer at $\mathrm{pH} 7.2$ with $20 \mathrm{mM} \mathrm{NaCl}$ for $1 \mathrm{~h}$ at room temperature and then washed with plenty of $\mathrm{H}_{2} \mathrm{O}$. After that, it was allowed to equilibrate for $10 \mathrm{~min}$ in solutions of different concentrations of Gal-3 ranging from 0 to $500 \mathrm{nM}$. Nitrogen was bubbled through the solutions for 3 min before each measurement. Differential pulse voltammetry experiments were measured with a scan rate of $5 \mathrm{mVs}^{-1}$, a step potential of $20 \mathrm{mV}$, a modulation amplitude of $50 \mathrm{mV}$, a modulation time of $0.05 \mathrm{~s}$, and an interval time of $2 \mathrm{~s}$ between 0 and $1 \mathrm{~V}$ for AuNP@Fc-Lac, 0 and $0.8 \mathrm{~V}$ for dendrimers 12-14, and 0 and 0.7 for 18. 


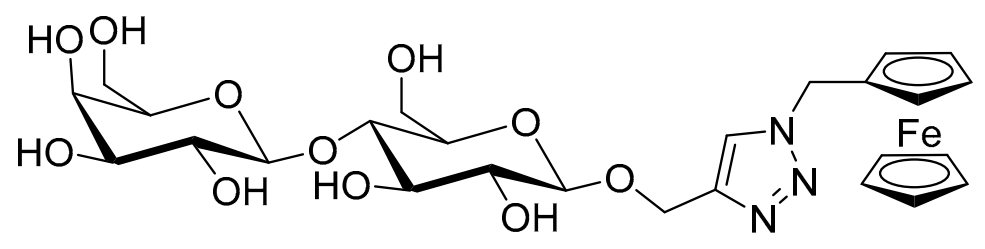

18

Figure 1. Chemical structure of compound 18.

\subsection{Isothermal Titration Calorimetry Measurements}

Calorimetric experiments were conducted using either an MCS or an ultrasensitive VP-ITC (Microcal Inc., Northampton, MA, USA). The preparation of samples and isothermal titration calorimetry (ITC) experiments were carried out as previously described elsewhere [50]. Titrations were routinely performed in a $20 \mathrm{mM}$ phosphate buffer at $\mathrm{pH} 7.2$ with $150 \mathrm{mM} \mathrm{NaCl}$ at $25^{\circ} \mathrm{C}$. During titrations, the reaction mixture was continuously stirred at $370 \mathrm{rpm}$ and the reference cell was filled with Milli-Q water. Blank titrations of ligand into buffer were also performed to correct the heat generated by dilution and mixing. The amount of heat produced per injection was calculated by integration of the individual peaks by the Origin software provided with the instrument. An equal and independent site model was used to fit the experimental data. This model provided the binding constant, the enthalpy change, and the number of binding sites (n) along with the corresponding standard deviations. The changes in the standard free energy $\Delta G^{0}$ and entropy $\Delta S^{0}$ were determined as $\Delta G^{0}=-\mathrm{R} T \ln K$ and $\mathrm{T} \Delta S^{0}=\Delta H-\Delta G^{0}$ (assuming that $\Delta H=\Delta H^{0}$ ).

\section{Results and Discussion}

\subsection{Synthesis}

To generate multivalent presentations of ferrocene-lactose (Fc-Lac) conjugates, we chose PAMAM dendrimers and Au nanoparticles (AuNPs) as scaffolds for the display of the conjugates. We started our studies with the preparation of building block 1, consisting of an azide-functionalized lactose-ferrocene conjugate, which is ready for further conjugation or direct coupling using azide-alkyne cycloaddition (AAC). This building block 1 was key for the convenient synthesis of the multivalent electrochemical probes. Commercially available 1,1'-bis(hydroxymethyl)ferrocene 2 was regioselectively transformed, in one step, into mono-azidoferrocene 3 in high a yield (Scheme 1) [36]. Cu(I)-catalyzed AAC of ferrocene derivative 3 with propargyl $\beta$-D-lactoside 4 [45] afforded Fc-Lac conjugate 5 in an almost quantitative yield. The treatment of such conjugate with sodium azide in aqueous $\mathrm{HCl}$ allowed the introduction of a new azido group in a high yield. The resulting azido-functionalized Fc-Lac 1 was then used for an additional Cu (I)-catalyzed AAC with alkyne-terminated PAMAM dendrimers 9-11 to obtain Fc-Lac dendrimers 12-14 in 78-81\% yields. Similarly, azido-functionalized Fc-Lac 1 reacted with alkynylated disulfide 16 [36] to obtain Fc-Lac disulfide $\mathbf{1 7}$ in a 95\% yield. Alkyne-terminated PAMAM dendrimers 9-11 and disulfide $\mathbf{1 6}$ were prepared via the reaction of the corresponding amino derivatives 6-8 and 15 with pent-4-ynoic anhydride [51] (Scheme 1). 


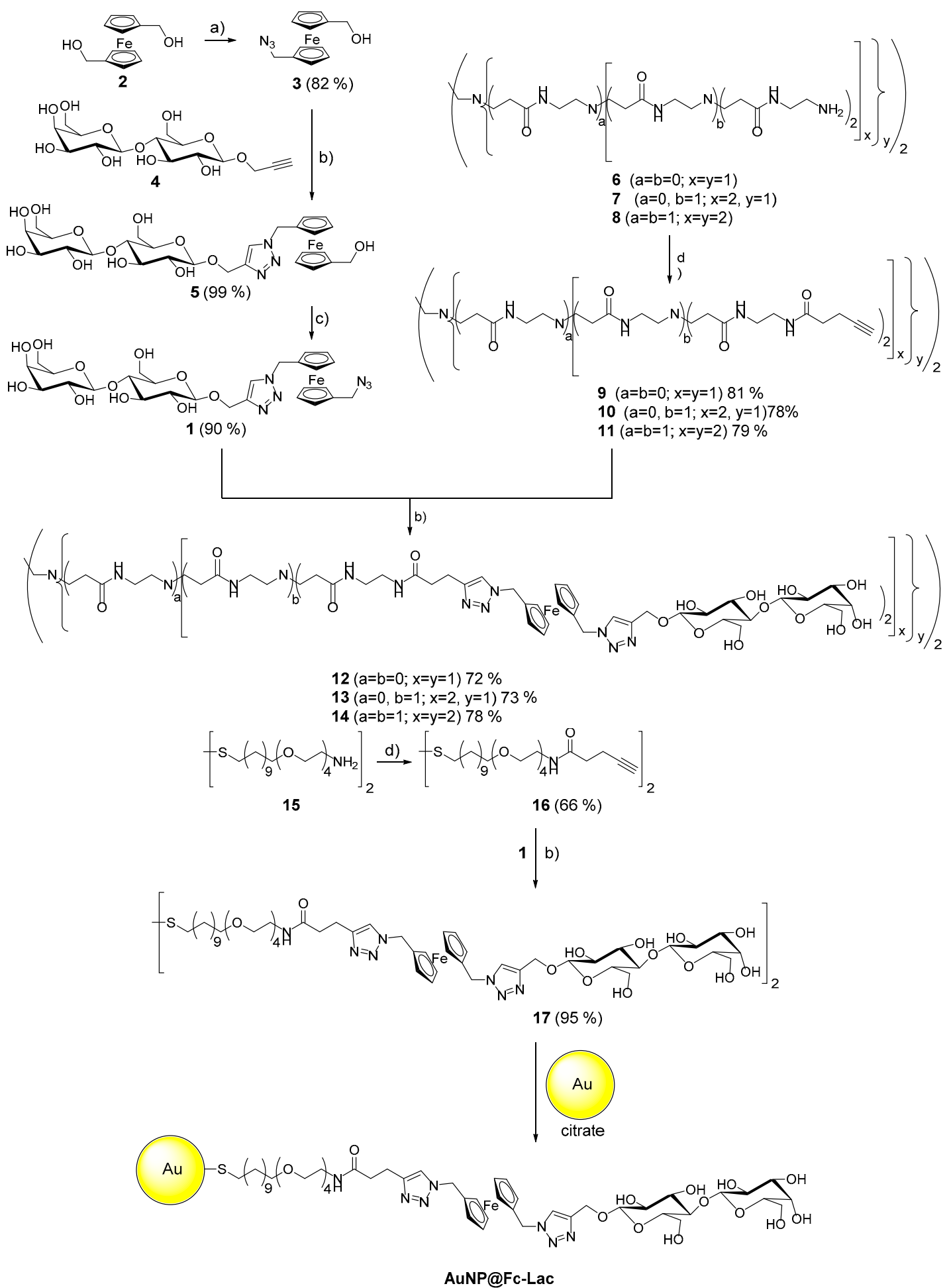

Scheme 1. (a) $\mathrm{NaN}_{3}, \mathrm{HCl}$, EtOAc/ $\mathrm{H}_{2} \mathrm{O}$, room temperature, $45 \mathrm{~min}$; (b) $\mathrm{CuSO}_{4}$, sodium ascorbate, $\mathrm{THF} / \mathrm{H}_{2} \mathrm{O}$, room temperature, overnight; (c) $\mathrm{NaN}_{3}, \mathrm{HCl}_{(\mathrm{aq})}$, room temperature, $2 \mathrm{~h}$; (d) pent-4-ynoic anhydride, $\mathrm{CH}_{2} \mathrm{Cl}_{2} / \mathrm{MeOH}$, room temperature, overnight.

The ${ }^{1} \mathrm{H}$ - and ${ }^{13} \mathrm{C}-\mathrm{NMR}$ analysis of Fc-Lac dendrimers $\mathbf{1 2 - 1 4}$ and disulfide $\mathbf{1 7}$ evidenced the presence of two chemically different 1,2,3-triazole rings. The ${ }^{1} \mathrm{H}$ NMR spectra revealed two singlets at 8.06-8.08 and 7.75-7.76 ppm, corresponding to the 1,2,3-triazole methine protons. The ${ }^{13} \mathrm{C}$ NMR spectra displayed four signals: two at 146.0 and $143.7 \mathrm{ppm}$ and two at 123.7 and 121.4, corresponding 
to the triazole C-4 and C-5 atoms, respectively. The ${ }^{13} \mathrm{C}$ NMR spectra also showed two signals at 103.8-103.9 and 101.8-101.9 ppm corresponding to the anomeric carbons of the $\beta$-D-galacto- and $\beta$-D-glucopyranoside units of the $\beta$-D-lactoside moieties. In addition, the protons of the methylenes linking the triazole moieties to the metallocene units were clearly observable in the ${ }^{1} \mathrm{H}$ NMR spectra as two singlets at 5.32-5.32 and 5.27-5.26 ppm. The ESI- and MALDI-TOF spectra confirmed the molecular weight of Fc-Lac dendrimers 12-14 and disulfide 17. For the Fc-Lac dendrimers, the number of carbohydrate units per dendrimer was determined by means of sulfuric acid-phenol assays to be 4.1 $\pm 0.2,8.0 \pm 0.2$ and $16.4 \pm 1.1$ for $\mathbf{1 2}, \mathbf{1 3}$, and 14, respectively (see Supplementary Materials) [38].

Fc-Lac disulfide 17 was employed to functionalize citrated-capped AuNPs. Citrate-capped gold nanoparticles were prepared according to a modification [46] of the Turkevich protocol [47], as reported elsewhere [36,45,52]. Functionalized AuNPs were prepared by mixing an excess of Fc-Lac disulfide 17 with $12 \mathrm{~nm}$ citrate-capped AuNPs in $1 \mathrm{mM} \mathrm{NaOH}$ for $24 \mathrm{~h}$ at room temperature. After removing the unreacted disulfide 17, the UV/Vis spectra of the resulting Fc-Lac-functionalized AuNPs (AuNPs@Fc-Lac) revealed a bathochromic shift of $8 \mathrm{~nm}$ of the SPR band. Redshift is a distinct indication of sulfur anchoring and has been attributed to changes in the electronic environment on the surface of AuNPs $[36,45,53,54]$. No broadening in the SPR band was observed, which excluded ligand-induced AuNPs aggregation. The average diameter $(12.2 \mathrm{~nm})$ of the nanoparticle gold cores was unaltered after functionalization, as determined by TEM analyses (see Supplementary Materials for details). The average number of ligands per nanoparticle was estimated to be $1765 \pm 32$ based on the elemental gold-to-iron ratio obtained by inductively coupled plasma optical emission spectroscopy (ICP-OES) in combination with the TEM data (see Supplementary Materials)

\subsection{Electrochemical Characterization}

The differential pulse voltammograms of the Fc-Lac dendrimers 12-14 and mono-Fc-Lac conjugate 18 [32] in $10 \mathrm{mM}$ phosphate buffer at pH 7.2 with $20 \mathrm{mM} \mathrm{NaCl}$ as the supporting electrolyte are shown in Figure 2. The voltammograms of multivalent compounds 12-14 showed only one peak potential $\left(E_{\mathrm{p}}\right)(+0.456,+0.461$, and $+0.482 \mathrm{~V}$, respectively) for the oxidation of the ferrocene moieties (Table 1), which is consistent with the apparent equivalence of all ferrocene residues displayed by each glycodendrimer at the timescale of the electrochemical measurement $[38,55]$. Contrary to the previously investigated mannosylferrocenyl PAMAM dendrimers [38], in which the observed oxidation potential was independent of the dendrimer generation, we observed a slight shift to a more positive $E_{\mathrm{p}}$ value of $-0.026 \mathrm{~V}$ when going from dendrimer generation G0 to G2. This behavior may be related to the lesser polar microenvironment around the ferrocene residue with an increase of dendrimer generation. Moving from a monosaccharide to a disaccharide may lead to a more shielded ferrocene from solvent interactions and will, therefore, make the ferrocene moiety more difficult to oxidize. This shielding effect would be more effective in dendrimer 14 with the highest generation. Another variation from the previously reported glycoferrocenyl dendrimer is the substitution of an electron-withdrawing amide group directly attached to the ferrocenyl group by an electron-donating methyltriazole group, which led to lower $E_{\mathrm{p}}$ values than those reported $(+0.560 \mathrm{~V})$. 


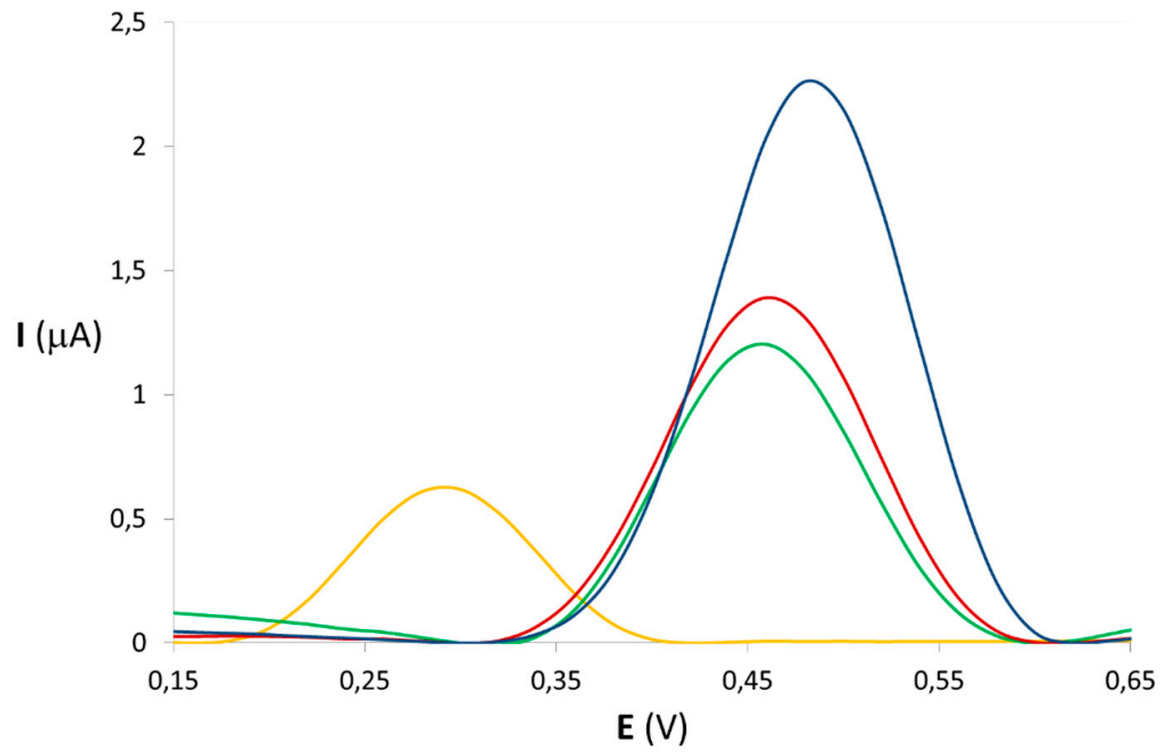

Figure 2. Differential pulse voltammograms for $12(25 \mu \mathrm{M}$, green), $13(12.5 \mu \mathrm{M}$, red), $14(6.25 \mu \mathrm{M}$, blue), and $18\left(50 \mu \mathrm{M}\right.$, orange) in $10 \mathrm{mM}$ phosphate buffer at $\mathrm{pH} 7.2$ and $25^{\circ} \mathrm{C}$ with $20 \mathrm{mM} \mathrm{NaCl}$ as the supporting electrolyte.

Table 1. Oxidation potential $\left(E_{\mathrm{p}}\right)$ and current intensity $\left(I_{\mathrm{p}}\right)$ of differential pulse voltammetry (DPV) peaks for 18, and 12-14 in $10 \mathrm{mM}$ phosphate buffer at $\mathrm{pH} 7.2$ and $25^{\circ} \mathrm{C}$ with $20 \mathrm{mM} \mathrm{NaCl}$.

\begin{tabular}{ccccc}
\hline Compound & Concentration $(\mu \mathbf{M})$ & Ferrocene Concentration $(\mu \mathrm{M})$ & $E_{\mathbf{p}}(\mathbf{V})$ & $I_{\mathbf{p}}(\mu \mathrm{A})$ \\
\hline $\mathbf{1 8}$ & 50 & 50 & +0.292 & 0.62 \\
$\mathbf{1 2}$ & 25 & 100 & +0.456 & 1.21 \\
$\mathbf{1 3}$ & 12.5 & 100 & +0.461 & 1.39 \\
$\mathbf{1 4}$ & 6.25 & 100 & +0.482 & 2.26 \\
\hline
\end{tabular}

Interestingly, the DPV peak current $\left(I_{\mathrm{p}}\right)$ values $0.62,1.21,1.39$, and $2.26 \mu \mathrm{A}$ for mono- 18, tetra- 12, octa- 13, and hexadecamer 14, respectively, increase with the glycodendrimer generation (Table 1). This trend is the opposite of that observed for the previously reported mannosylferrocenyl dendrimer [38]. The $I_{\mathrm{p}}$ value is directly proportional to the concentration and the square of the diffusion coefficient of the electroactive species, the number of exchanged electrons, and the electrode surface [56]. Hence, for lactosylferrocenyl dendrimers, the increase of the electrochemical signal that results from the increased number of exchanged electrons prevails over the expected decrease of the peak current due to the slower diffusion of the larger electroactive species. This behavior might be due to stronger adsorption of the larger dendrimer onto the electrode, which results in an enhancement of the $I_{\mathrm{p}}$ value [55].

The electrochemical properties of AuNP@Fc-Lac were also studied by DPV. DPV measurements of AuNP@Fc-Lac were carried out on a $20 \mathrm{nM}$ nanoparticle solution in $10 \mathrm{mM}$ phosphate buffer at $\mathrm{pH} 7.2$ with $20 \mathrm{mM} \mathrm{NaCl}$, after being incubated for $1 \mathrm{~h}$ in the presence of a glassy carbon working electrode, an $\mathrm{Ag} / \mathrm{AgCl}(3 \mathrm{M} \mathrm{KCl})$ reference electrode, and a Pt sheet counter electrode. The differential pulse voltammogram of AuNP@Fc-Lac showed an oxidation peak with a peak current value of $6.11 \mu \mathrm{A}$ at an oxidation potential value of $0.620 \mathrm{~V}$.

\subsection{Binding Abilities Toward Galectin-3}

We investigated the binding abilities of mono- 18, tetra- 12, octa- 13, and hexadeca-Fc-Lac 14 toward Gal-3, as we envisaged that enhanced Gal-3 binding due the multivalent effect would contribute to improved sensing abilities. 
Isothermal titration calorimetry (ITC) provided the thermodynamic parameters $\left(K, \Delta G^{0}, \Delta H\right.$, and $\mathrm{T} \Delta S^{0}$ ) that allow us to characterize how multivalency affects the binding affinities. ITC experiments were developed at pH 7.2 in $20 \mathrm{mM}$ phosphate buffer with $150 \mathrm{mM} \mathrm{NaCl}$ at $25^{\circ} \mathrm{C}$. The stepwise addition of a solution of Fc-Lac 18 and 12-14 into a solution of Gal-3 led to a series of exothermic peaks with a decreasing area. Figure 3 shows a representative thermogram for the binding of 13 to Gal-3. Calorimetric data were fitted to an equal and independent binding site model facilitating the direct determination of the data displayed in Table 2. Globally, the complexation of the Fc-Lac derivatives with Gal-3 is enthalpy driven and entropically unfavorable, as the binding is dominated by enthalpy contributions (Figure 4).

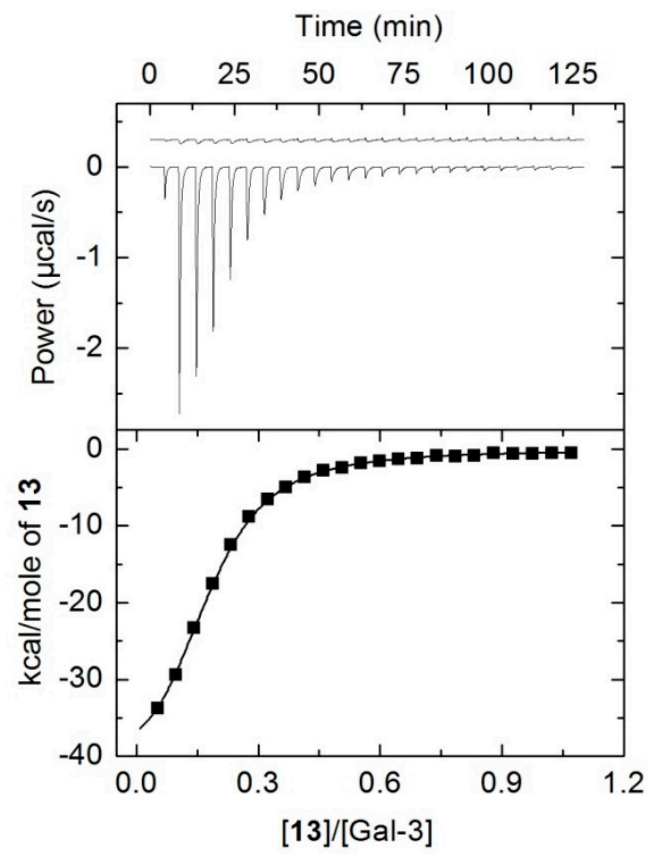

Figure 3. Representative titration of galectin-3 (Gal-3) $(40.58 \mu \mathrm{M})$ with compound $\mathbf{1 3}(0.514 \mathrm{mM})$ in 20 $\mathrm{mM}$ phosphate buffer at $\mathrm{pH} 7.2$ with $150 \mathrm{mM} \mathrm{NaCl}$ at $25^{\circ} \mathrm{C}$. The top panel shows the raw calorimetric data for 25 automatic injections, $5 \mu \mathrm{L}$ each $(1 \mu \mathrm{L}$ for the first) of 13 . The area under each peak represents the amount of heat released upon binding of the conjugate to the lectin. The smooth solid lines (bottom panel) represent the best fit of the experimental data to a model of $n$ equal and independent sites.

Table 2. Thermodynamic parameters for compounds 12-14 and $\mathbf{1 8}$ obtained by isothermal titration calorimetry in $20 \mathrm{mM}$ phosphate buffer at pH 7.2 with $150 \mathrm{mM} \mathrm{NaCl}$ at $25^{\circ} \mathrm{C}$.

\begin{tabular}{|c|c|c|c|c|c|}
\hline Compound & $\begin{array}{c}-\Delta \mathrm{G}^{0} \\
\left(\mathrm{~kJ} \cdot \mathrm{mol}^{-1}\right)\end{array}$ & $\begin{array}{c}-\Delta \mathrm{H} \\
\left(\mathrm{kJ} \cdot \mathrm{mol}^{-1}\right)\end{array}$ & $\begin{array}{c}\mathrm{T} \Delta \mathrm{S}^{0} \\
\left(\mathrm{~kJ} \cdot \mathrm{mol}^{-1}\right)\end{array}$ & $\mathrm{K} \cdot 10^{-4}\left(\mathrm{M}^{-1}\right)$ & n \\
\hline 18 & $21.74 \pm 0.11$ & $37.81 \pm 3.72$ & $-16.07 \pm 3.70$ & $0.66 \pm 0.03$ & 1.00 \\
\hline 12 & $31.48 \pm 0.14$ & $194.66 \pm 7.30$ & $-163.18 \pm 7.25$ & $31.82 \pm 2.10$ & $0.19 \pm 0.05$ \\
\hline 13 & $32.90 \pm 0.06$ & $194.83 \pm 2.01$ & $-161.93 \pm 1.98$ & $54.82 \pm 1.40$ & $0.17 \pm 0.01$ \\
\hline 14 & $34.36 \pm 0.31$ & $97.02 \pm 3.60$ & $-62.66 \pm 3.20$ & $103.04 \pm 13.0$ & $0.26 \pm 0.01$ \\
\hline
\end{tabular}




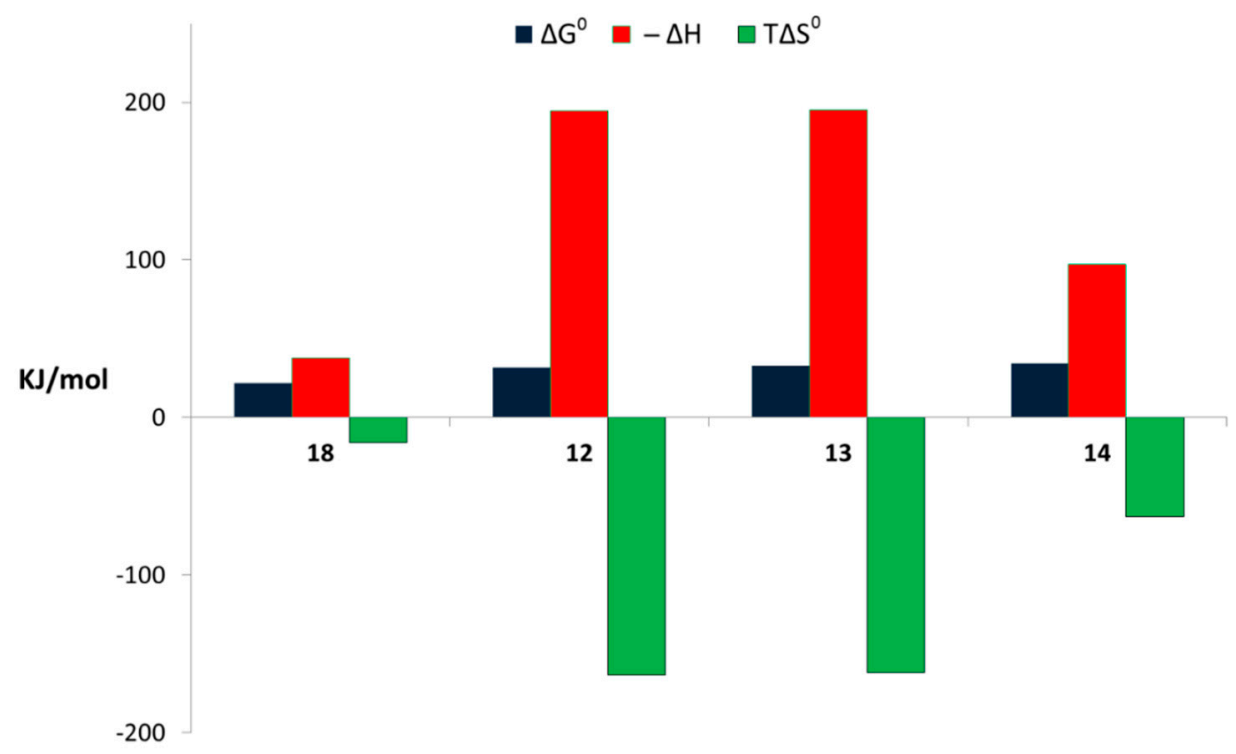

Figure 4. Free energy $\left(-\Delta G^{0}\right.$, black), enthalpy $\left(-\Delta H\right.$, red), and entropy changes $\left(T \Delta S^{0}\right.$, green) for the binding of Fc-Lac dendrimers 12-14 and monovalent glycoconjugate 18 to Gal-3 obtained from isothermal titration calorimetry (ITC) experiments in $20 \mathrm{mM}$ phosphate buffer at pH 7.2 with $150 \mathrm{mM}$ $\mathrm{NaCl}$ at $25^{\circ} \mathrm{C}$.

As can be seen in Table 2, the $K$ values increase with the number of lactoside residues of the dendrimers, from monomer 18 to hexadecamer 14, due to a multivalent effect, with the binding affinities of tetramer 12, octamer 13, and hexadecamer 14 being -48-, -83-, and -156-fold higher than that for monomer 18. A deeper analysis revealed that binding enthalpy $\Delta H$ values rapidly decrease (become more favorable) when going from monomer 18 to dendrimer 12, which possesses four lactoside residues, where the $\Delta H$ value of $\mathbf{1 2}$ is -5 times lower than that for $\mathbf{1 8}$. However, moving from tetramer 12 to octamer 13 , a small $\Delta \Delta H$ value of $-0.17 \mathrm{~kJ} \mathrm{~mol}^{-1}$ is observed, which likely means that not all the lactoside moieties are involved in the binding. Furthermore, the increase in the multivalence to 16 lactoside residues led to an increase of $97.81 \mathrm{~kJ} \mathrm{~mol}^{-1}$ (less favorable) of the enthalpy binding. Nevertheless, these changes in enthalpy binding with an increase in the number of lactoside residues are counterbalanced by changes in unfavorable entropic contributions. For example, a less favorable enthalpy binding for 16-mer 14, compared to 4-mer 12 and 8-mer 13, is compensated with a less unfavorable entropic term than that for the compounds. Thus, a strong enthalpy-entropy compensation behavior is observed with a slope slightly greater than unity (Figure 5), which is typically found in carbohydrate-lectin binding interactions [57,58]. Although the origin of this phenomenon remains unclear, such compensatory behavior has been attributed to solvent reorganization upon ligand binding, suggesting that the Fc-Lac conjugates follow a similar binding mechanism. The $n$ values are lower than unity for all multivalent glycoconjugates (Table 2), as expected for the interactions of multivalent carbohydrates with oligomeric lectins [59]. Such behavior suggests an increase in the cross-linking between the glycoconjugates and Gal-3, with an increase of the number in lactoside residues. However, while Fc-Lac dendrimers 12 and 13 showed $n$ values below and relatively close to the theoretically expected values for a tetramer $(1 / 2=0.25)$ and an octamer $(1 / 8=0.125)$, Fc-Lac 16-mer 14 showed a value larger than that theoretically expected $(1 / 16=0.063)$, which indicates that, for this compound, not all of the lactoside residues participate in the binding, as noted above. 


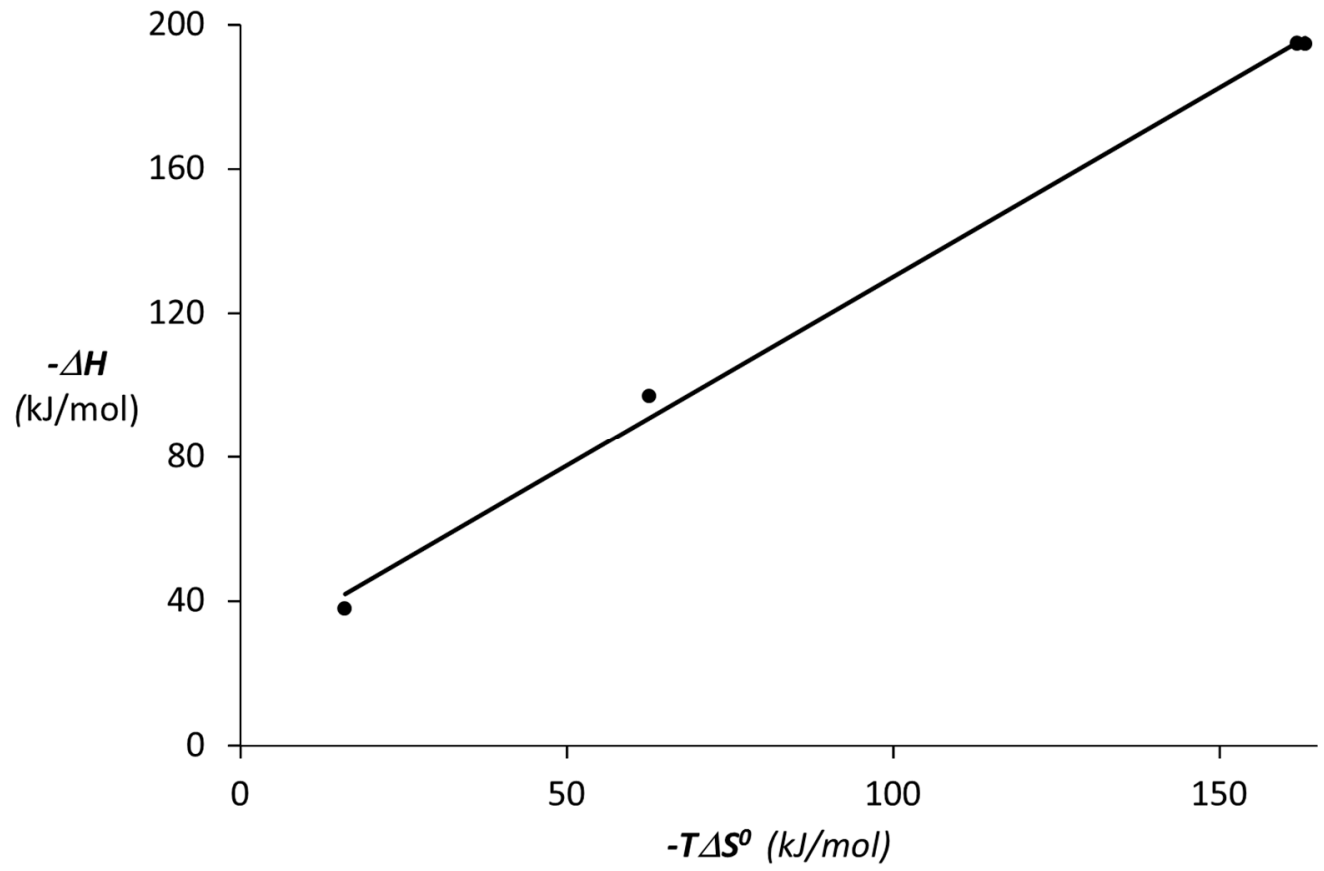

Figure 5. Enthalpy-entropy compensation plot. Plot of the $-\Delta H$ versus $-T \Delta S^{0}$ for the binding of compounds 12-14 and $\mathbf{1 8}$ to Gal-3. The plot shows a linear relationship with a slope of 1.05 and a correlation coefficient of 0.997 .

It is noteworthy to mention that the binding affinity of Fc-Lac dendrimers, and particularly hexadecamer 14, is within the range of magnitude of the potent Gal-3 inhibitors [4,60]. Therefore, they can be applied as anti-Gal-3 agent for therapeutic purposes. We tested the binding ability of AuNP@Fc-Lac toward Gal-3 by following their UV-vis spectra variations after the addition of the lectin. It is well-known that this technique is a very useful tool to demonstrate protein-induced aggregation of AuNPs due to the dependence of the surface plasmon resonance (SPR) band on interparticle distance [36,61,62]. Thus, upon AuNPs aggregation, coupling interactions between the surface plasmon fields of the nanoparticles take place, leading to a change of the local refractive index around the nanoparticles, a red shift of the SPR band, and (usually) a broadening that, on occasion, results in absorbance decay.

The treatment of the AuNP@Fc-Lac solution with Gal-3 in a phosphate buffer at pH 7.2 led to a red shift of the SPR band along with a progressive decrease of its absorbance $\left(A_{\mathrm{SPR}}\right)$ with time (Figure 6$)$. In this experiment, the used solution contained $20 \mathrm{mM} \mathrm{NaCl}$ in order to keep a high ionic strength and to ensure the stability of the protein and avoid its aggregation. Both the SPR band increase $\left(\Delta \lambda_{\mathrm{SPR}}\right)$ and absorbance decrease $\left(\triangle A_{\mathrm{SPR}}\right)$ versus when time plots reached a plateau at $90 \mathrm{~min}$. These results can be reasoned in terms of the closer special arrangement of AuNPs as a result of the formation of cross-linked complexes between AuNP@Fc-Lac and Gal-3. Thus, an increase in size of the aggregates with time brings about both a shift and a broadening of the SPR band. Eventually, the complex became water-insoluble and precipitated, leading to a decrease in absorbance. This UV-vis behavior of the binding interaction of Gal-3 and AuNP@Fc-Lac contrasts with that observed for the AuNP@Lac without a ferrocene moiety, which exhibited, in the presence of five times more concentrated Gal-3, a negligible change of $\lambda_{\mathrm{SPR}}$ and an increase of $A_{\mathrm{SPR}}$ during a period of $5 \mathrm{~h}$ [45]. Either the presence of the ferrocene moiety or the lower Gal-3 concentration at which the experiments were carried out (or both) seem to favor faster aggregation upon binding interactions, and allow for an arrangement of the AuNPs at more optimal interparticle distances. To test the participation of the lactose moiety in the binding, a saturated aqueous solution of lactose was added to the sample containing AuNP@Fc-Lac and Gal-3 after a period of incubation of $1.5 \mathrm{~h}$. After $15 \mathrm{~min}$, a completed re-dissolution was observed and the UV-vis spectrum showed the recovery of the original SPR band, albeit at a lower absorbance than the 
original. Moreover, the UV-vis spectrum of AuNP@Fc-Lac in the presence of bovine serum albumin (BSA) under the same conditions did not show any SPR band shift after a period of incubation of $5 \mathrm{~h}$ (Figure 6). These results confirm the specificity of the binding interaction and the lack of significance of the non-specific interactions.
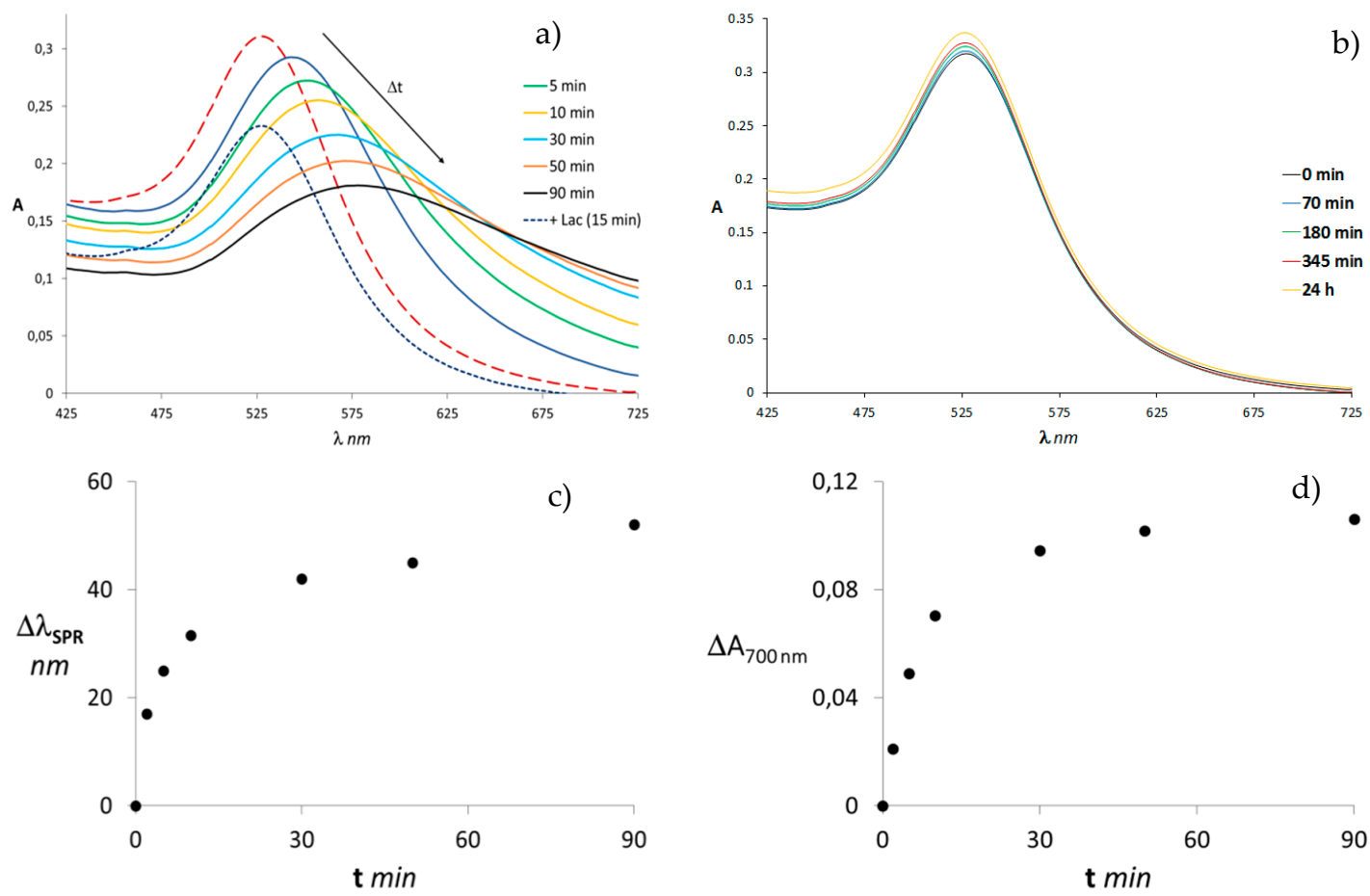

Figure 6. (a) UV-visible spectra of AuNP@Fc-Lac $(2 \mathrm{nM})$ in a phosphate buffer of $10 \mathrm{mM}$, pH 7.2 with 20 $\mathrm{mM} \mathrm{NaCl}$ in the presence of Gal-3 $(10 \mu \mathrm{M})$ recorded at different times after addition of the lectin along with the UV-visible spectrum of this mixture after the addition of an aqueous saturated D-Lactose solution (dashed blue). (b) UV-visible spectra of AuNP@Fc-Lac (2 nM) in phosphate buffer $10 \mathrm{mM}, \mathrm{pH}$ 7.2 , in the presence of BSA recorded at different times. Variation of $\Delta \lambda_{\mathrm{SPR}}$ (c) and absorbance at $700 \mathrm{~nm}$ (d) over time.

\subsection{Sensing Abilities Toward Gal-3}

Once we had demonstrated the redox properties of PAMAM@Fc-Lac 12-14 and AuNP@Fc-Lac and their strong specific binding interactions with Gal-3, we studied their electrochemical sensing properties. In order to assess such sensing abilities, we evaluated the extent of the variation of the peak current upon the interaction of the electroactive spices with Gal-3. For this assessment, we used sensitivity parameters $\left(P_{\mathrm{s}}\right)$, which are defined as $\left(I_{\mathrm{p} 0}-I_{\mathrm{p}}\right) / I_{\mathrm{p} 0}$, where $I_{\mathrm{p} 0}$ and $I_{\mathrm{p}}$ represent the peak currents in the absence and in the presence of Gal-3, respectively. The $P_{\mathrm{s}}$ parameter is proportionally related to the number of redox units that became incorporated into the low-diffusing cross-linked complex upon binding with the lectin. Consequently, this parameter is related to both the stability of the complexes ( $K$ values) and the number of ferrocene units (valency) participating in the complex. In all cases, DPV voltammograms showed a progressive decrease of the $I_{\mathrm{p}}$ values as the Gal-3 concentration increased, which is indicative of the formation of electroactive Fc-Lac conjugate-Gal-3 complexes (Figures 7 and 8 ). As the $I_{\mathrm{p}}$ value is proportional to the square root of the diffusion coefficient of the electroactive species, and the Fc-Lac-conjugate-Gal-3 complexes are expected to diffuse slower than the free Fc-Lac conjugate, the complexes have smaller $I_{\mathrm{p}}$ values than the uncomplexed species. 

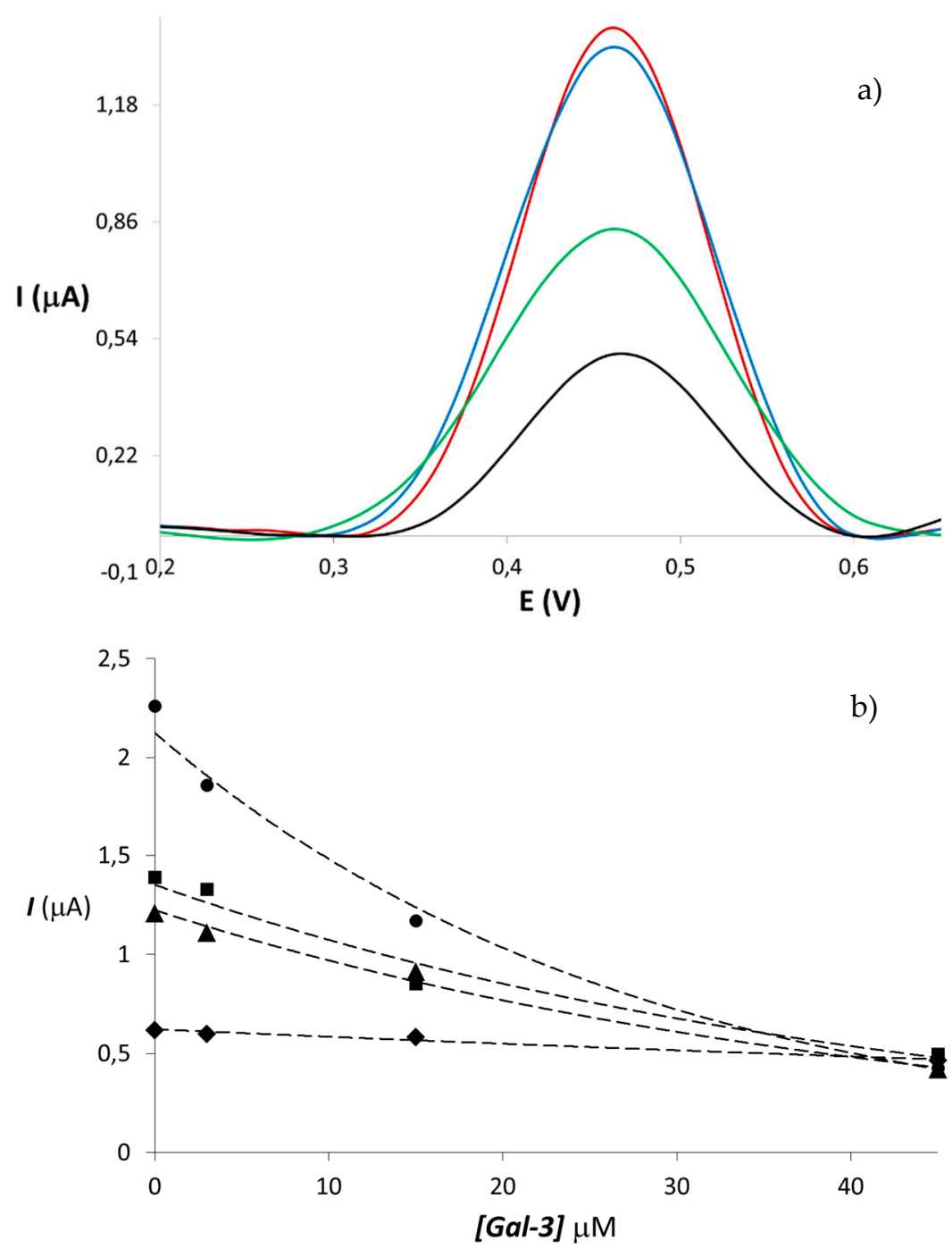

Figure 7. (a) Differential pulse voltammograms for compound $13(12.5 \mu \mathrm{M})$ in the presence of increasing amounts of Gal-3 $(0 \mu \mathrm{M}$, red; $3 \mu \mathrm{M}$, blue; $15 \mu \mathrm{M}, 45 \mu \mathrm{M}$ ) in $10 \mathrm{mM}$ phosphate buffer at pH 7.2 with 20 $\mathrm{NaCl}$. A decrease in the peak current was observed as the Gal-3 concentration increased. (b) Variation

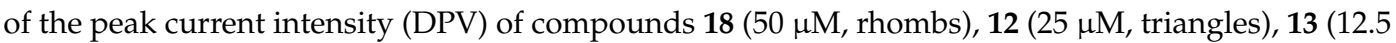
$\mu \mathrm{M}$, squares), and $14(6.25 \mu \mathrm{M}$, circles) in the presence of increasing amounts of Gal-3 (dashed lines represent the exponential fits).

The $P_{\mathrm{S}}$ of the Fc-Lac conjugates is shown in Figure 9. The highest sensitivity to Gal-3 is shown by AuNP@Fc-Lac, which is able to detect the lectin in nanomolar concentrations. The estimated limit of detection (LOD) for AuNP@Fc-Lac was calculated to be $-160 \mathrm{nM}$, which is one and two orders of magnitude lower than that for PAMAM@Fc-Lac derivatives 12-14 and monomer Fc-Lac 18, respectively (Figure 10). A $20 \mathrm{nM}$ concentration of AuNP@Fc-Lac is slightly more sensitive than a $6.25 \mu \mathrm{M}$ concentration of 16-mer 14 to detect Gal-3 at a concentration 15 times lower. Nevertheless, it should be noted that unlike the DPV measurements for 18 and 12-14, those for AuNP@Fc-Lac involved electrode adsorption. Although the $P_{\mathrm{S}}$ values depend on the concentration of Gal-3, it can be observed that 16-mer 14 is the most sensitive PAMAM@Fc-Lac derivative in all tested Gal-3 concentrations. At a $45 \mu \mathrm{M}$ concentration of Gal-3, which is above the LOD of the non-metallic Fc-Lac probes, glycodendrimer 14, with 16 lactose units, is at a $6.25 \mu \mathrm{M}$ concentration, 1.2-fold more sensitive than 4-mer 12 and 8-mer 13 at 25 and $12.5 \mu \mathrm{M}$ concentrations, respectively. Likewise, the sensing ability of 16-mer 14 toward Gal-3, at the mentioned concentrations, is 3.2 times higher than that of the 
monovalent derivative in a solution eight times more concentrated. At lower $15 \mu \mathrm{M}$ concentrations of Gal-3, the ratios of the $P_{\mathrm{s}}$ values for 16-mer $\mathbf{1 4}$ and 8-mer $\mathbf{1 3}$ are similar to those for a higher concentration of Gal-3, but the sensitivity of 4-mer $\mathbf{1 2}$ is -1.6 -fold and twofold lower than that for 8-mer 13 and 16-mer 14, respectively.

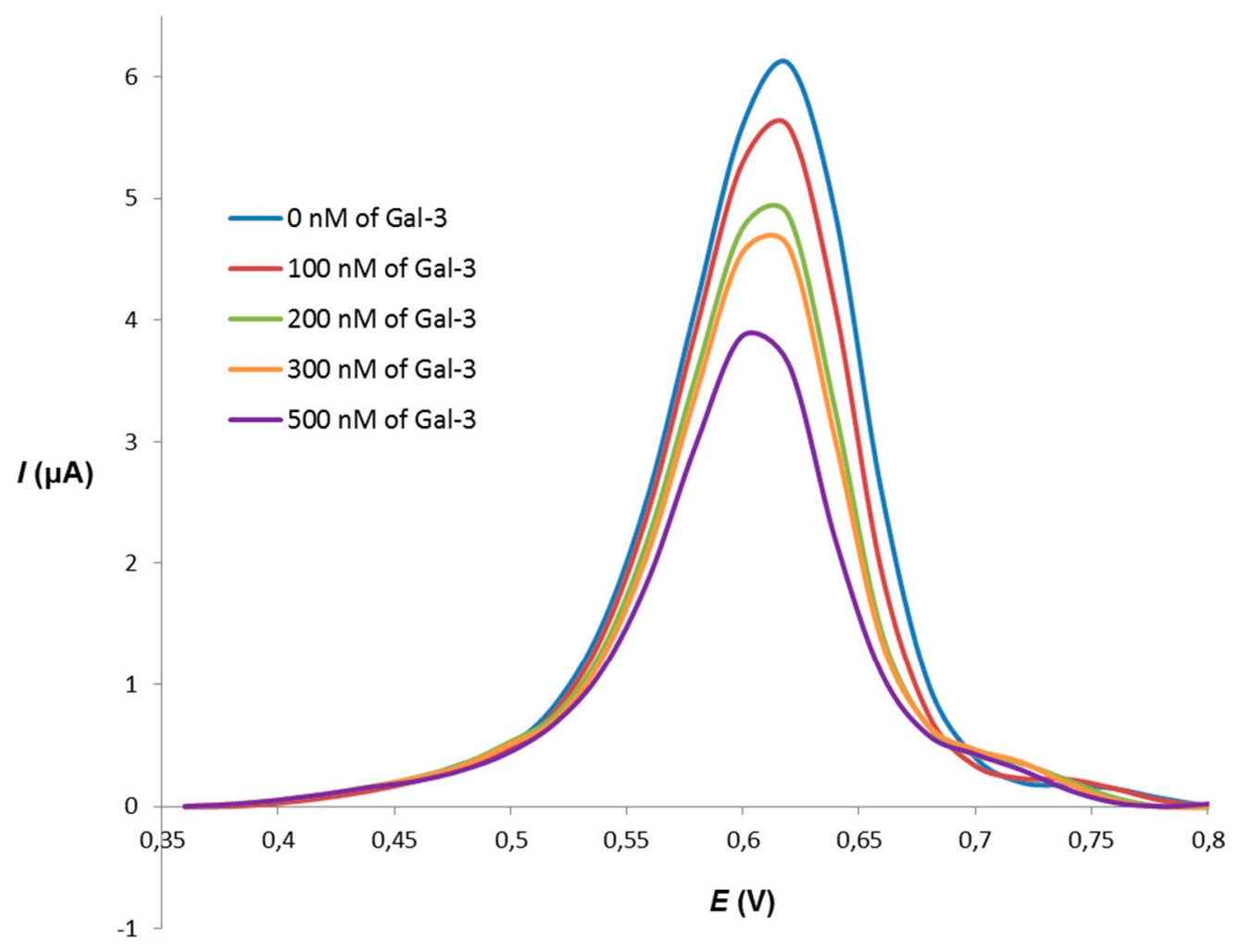

Figure 8. Differential pulse voltammogram for AuNP@Fc-Lac-modified glassy carbon electrode in the presence of increasing amounts of Gal-3 $(0 \mathrm{nM}$, blue; $100 \mathrm{nM}$, red; $200 \mathrm{nM}$, green; $300 \mathrm{nM}$, orange; 500 $\mathrm{nM}$, purple) in $10 \mathrm{mM}$ phosphate buffer at $\mathrm{pH} 7.2$ with $20 \mathrm{mM} \mathrm{NaCl}$ as the supporting electrolyte.

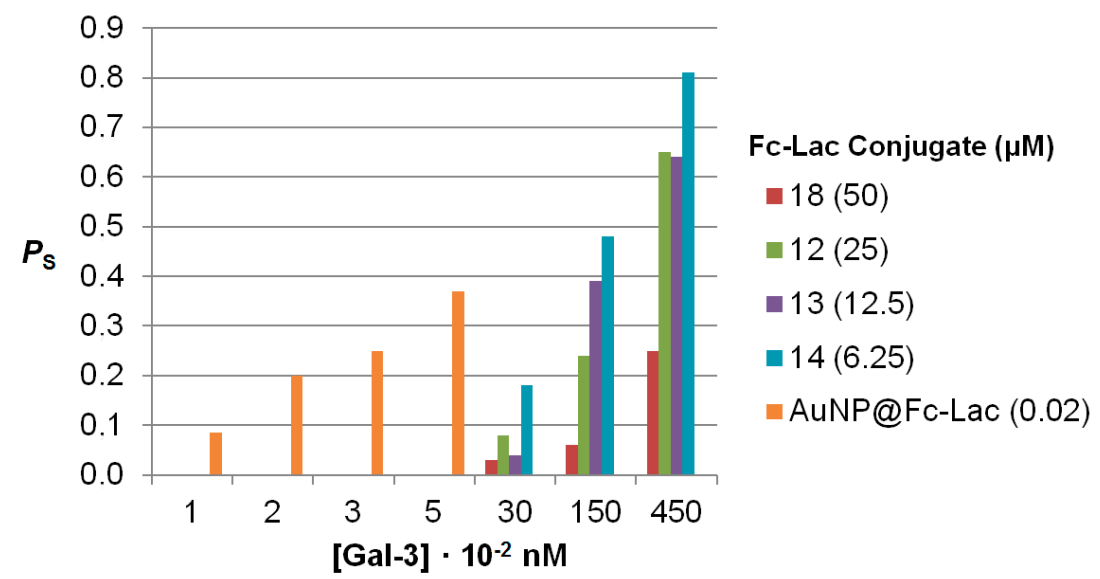

Figure 9. Sensitivity parameters $\left(P_{\mathbf{s}}\right)$ for ferrocene-lactose conjugates 12-14, 18, and AuNP@Fc-Lac with increasing concentrations of Gal-3. 


\section{Limit of Detection (LOD, nM)}

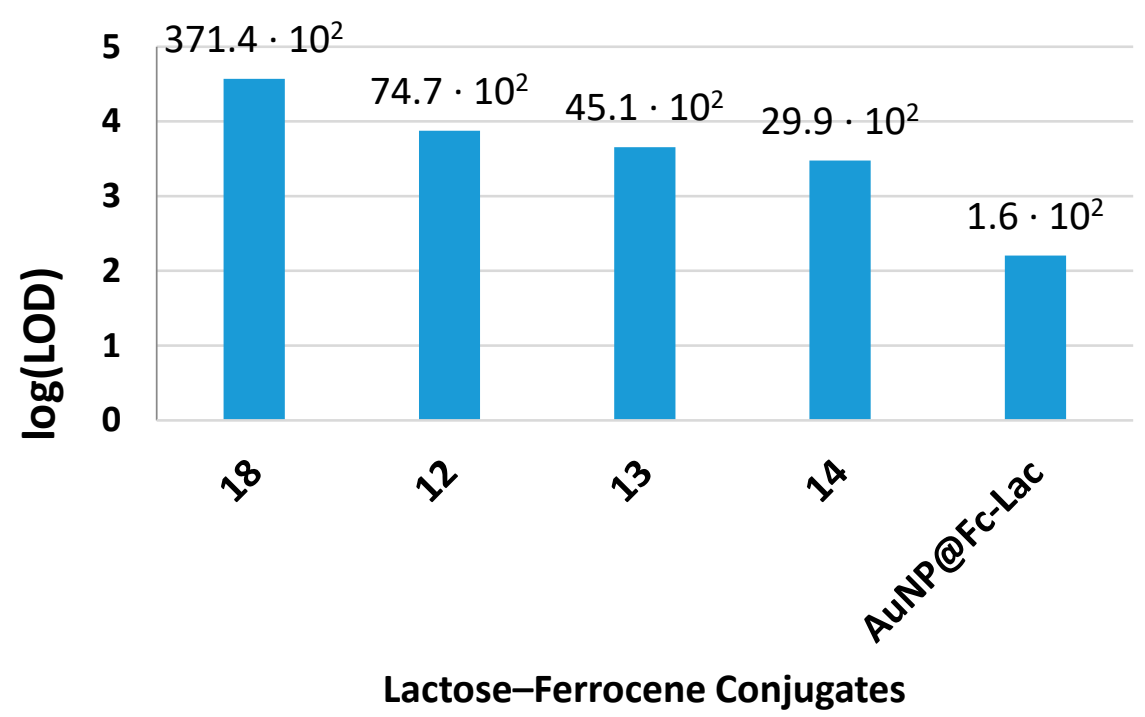

Figure 10. Limit of detection (LOD) for ferrocene-lactose conjugates 12-14, 18, and AuNP@Fc-Lac (logarithmic scale). The LOD (nM) value for each conjugate is shown over the respective bar.

\section{Conclusions}

We have developed simple and convenient methods for the preparation of electrochemical probes for the high sensitivity detection of galectin-3. These probes consist of multivalent presentations of lactose-ferrocene conjugates based on poly (amido amine) dendrimers and gold nanoparticles, enabling enhanced galectin-3 binding affinity and multielectron exchange, resulting in an important improvement of electrochemical sensing abilities. Both lactosylferrocenylated dendrimers and gold nanoparticles showed a high affinity toward galectin-3 by calorimetric and optical methods, respectively, demonstrating that they bind to the lectin through a multivalent interaction, despite the monomeric nature of the lectin. Interestingly, the affinity of the multivalent conjugates, particularly those with higher valency, is within the range of magnitude of the most potent galectin-3 inhibitors, therefore, they can be applied as therapeutic agents. The highest sensitivity to galectin-3 by electrochemical methods was shown by lactosylferrocenylated gold nanoparticles, which were able to detect the lectin in nanomolar concentrations at least one order of magnitude lower than that needed by the dendrimers.

Supplementary Materials: The following are available online at http://www.mdpi.com/2079-4991/10/2/203/s1, UV-visible spectra for AuNPs@Fc-Lac preparation; transmission electron microscopy analysis; isothermal titration calorimetry experiments for compounds 18, 12, and 14; differential pulse voltammetry titrations for compounds 18, 12, and 14 with galectin-3; sulfuric acid-phenol assays for compounds 12-14; sensitivity parameters for 12-14, 18, and AuNP@Fc-Lac; ICP-OES measurements; mass spectra for compounds 12-14 and 17; ${ }^{13} \mathrm{C}-\mathrm{NMR}$ and ${ }^{1} \mathrm{H}-\mathrm{NMR}$ spectra for compounds 12-14 and 17.

Author Contributions: Conceptualization, A.V.-B.; methodology, A.V.-B. and L.G.-F.; validation, A.V.-B. and L.G.-F.; formal analysis, L.G.-F. and M.C.M.-M.; investigation, M.C.M.-M. and I.Q.-S.; writing-original draft preparation, A.V.-B. and M.C.M.-M.; writing-review and editing, A.V.-B., L.G.-F., and M.C.M.-M.; supervision, A.V.-B. and L.G.-F.; project administration, A.V.-B.; funding acquisition, A.V.-B. All authors have read and agreed to the published version of the manuscript.

Funding: This research was funded by the Spanish Ministry of Economy and Competitiveness, grant number CTQ2017-90050-R. PhD scholarship of M.C.M.M. was funded by the Spanish Ministry of Education.

Conflicts of Interest: The authors declare no conflict of interest. 


\section{References}

1. Bojarová, P.; Křen, V. Sugared biomaterial binding lectins: Achievements and perspectives. Biomater. Sci. 2016, 4, 1142-1160. [CrossRef] [PubMed]

2. Keane, M.P. Galectin-3: Distant Biomarker or Relevant Target? Am. J. Respir. Crit. Care Med. 2016, 194, 7-9. [CrossRef] [PubMed]

3. Asleh, R.; Enriquez-Sarano, M.; Jaffe, A.S.; Manemann, S.M.; Weston, S.A.; Jiang, R.; Roger, V.L. Galectin-3 Levels and Outcomes After Myocardial Infarction: A Population-Based Study. J. Am. Coll. Cardiol. 2019, 73, 2286-2295. [CrossRef] [PubMed]

4. Téllez-Sanz, R.; García-Fuentes, L.; Vargas-Berenguel, A. Human Galectin-3 Selective and High Affinity Inhibitors. Present State and Future Perspectives. Curr. Med. Chem. 2013, 20, 2979-2990. [CrossRef]

5. Wang, L.; Guo, X.-L. Molecular regulation of galectin-3 expression and therapeutic implication in cancer progression. Biomed. Pharmacother. 2016, 78, 165-171. [CrossRef]

6. Paul, S.; Harshaw-Ellis, K. Evolving Use of Biomarkers in the Management of Heart Failure. Cardiol. Rev. 2019, 27, 153-159. [CrossRef]

7. Agnello, L.; Bivona, G.; Lo Sasso, B.; Scazzone, C.; Bazan, V.; Bellia, C.; Ciaccio, M. Galectin-3 in acute coronary syndrome. Clin. Biochem. 2017, 50, 797-803. [CrossRef]

8. Mueller, T.; Gegenhuber, A.; Leitner, I.; Poelz, W.; Haltmayer, M.; Dieplinger, B. Diagnostic and prognostic accuracy of galectin-3 and soluble ST2 for acute heart failure. Clin. Chim. Acta 2016, 463, 158-164. [CrossRef]

9. Mueller, T.; Egger, M.; Leitner, I.; Gabriel, C.; Haltmayer, M.; Dieplinger, B. Reference values of galectin-3 and cardiac troponins derived from a single cohort of healthy blood donors. Clin. Chim. Acta 2016, 456, 19-23. [CrossRef]

10. Pieters, R.J. Inhibition and detection of galectins. ChemBioChem 2006, 7, 721-728. [CrossRef]

11. Laaf, D.; Bojarová, P.; Pelantová, H.; Křen, V.; Elling, L. Tailored Multivalent Neo-Glycoproteins: Synthesis, Evaluation, and Application of a Library of Galectin-3-Binding Glycan Ligands. Bioconjug. Chem. 2017, 28, 2832-2840. [CrossRef] [PubMed]

12. Polat, V.; Bozcali, E.; Uygun, T.; Opan, S.; Karakaya, O. Diagnostic significance of serum galectin-3 levels in heart failure with preserved ejection fraction. Acta Cardiol. 2016, 71, 191-197. [CrossRef] [PubMed]

13. Zivicova, V.; Broz, P.; Fik, Z.; Mifkova, A.; Plzak, J.; Cada, Z.; Kaltner, H.; Kucerova, J.F.; Gabius, H.-J.; Smetana, K., Jr. Genome-wide Expression Profiling (with Focus on the Galectin Network) in Tumor, Transition Zone and Normal Tissue of Head and Neck Cancer: Marked Differences Between Individual Patients and the Site of Specimen Origin. Anticancer Res. 2017, 37, 2275-2288. [CrossRef] [PubMed]

14. Raspollini, M.R.; Luque, R.J.; Menendez, C.L.; Bollito, E.; Brunelli, M.; Martignoni, G.; Montironi, R.; Cheng, L.; Blanca, A.; Baroni, G.; et al. T1 high-grade bladder carcinoma outcome: The role of p16, topoisomerase-II $\alpha$, survivin, and E-cadherin. Hum. Pathol. 2016, 57, 78-84. [CrossRef] [PubMed]

15. Hoofnagle, A.N.; Wener, M.H. The fundamental flaws of immunoassays and potential solutions using tandem mass spectrometry. J. Immunol. Methods 2009, 347, 3-11. [CrossRef]

16. Jiang, W.; Yuan, R.; Chai, Y.-Q.; Yin, B. Amperometric immunosensor based on multiwalled carbon nanotubes/Prussian blue/nanogold-modified electrode for determination of $\alpha$-fetoprotein. Anal. Biochem. 2010, 407, 65-71. [CrossRef]

17. Tang, Z.; He, J.; Chen, J.; Niu, Y.; Zhao, Y.; Zhang, Y.; Yu, C. A sensitive sandwich-type immunosensor for the detection of galectin-3 based on N-GNRs-Fe-MOFs@AuNPs nanocomposites and a novel AuPt-methylene blue nanorod. Biosens. Bioelectron. 2018, 101, 253-259. [CrossRef]

18. Yoshioka, K.; Sato, Y.; Murakami, T.; Tanaka, M.; Niwa, O. One-Step Detection of Galectins on Hybrid Monolayer Surface with Protruding Lactoside. Anal. Chem. 2010, 82, 1175-1178. [CrossRef]

19. Primo, E.N.; Kogan, M.J.; Verdejo, H.E.; Bollo, S.; Rubianes, M.D.; Rivas, G.A. Label-Free Graphene Oxide-Based Surface Plasmon Resonance Immunosensor for the Quantification of Galectin-3, a Novel Cardiac Biomarker. ACS Appl. Mater. Interfaces 2018, 10, 23501-23508. [CrossRef]

20. Kamemura, K.; Kato, S. Detection of Lectins Using Ligand Blotting and Polyacrylamide-Type Glycoconjugate Probes. Anal. Biochem. 1998, 258, 305-310. [CrossRef]

21. Ballell, L.; Alink, K.J.; Slijper, M.; Versluis, C.; Liskamp, R.M.J.; Pieters, R.J. A New Chemical Probe for Proteomics of Carbohydrate-Binding Proteins. ChemBioChem 2005, 6, 291-295. [CrossRef] [PubMed] 
22. van Scherpenzeel, M.; Moret, E.E.; Ballell, L.; Liskamp, R.M.J.; Nilsson, U.J.; Leffler, H.; Pieters, R.J. Synthesis and Evaluation of New Thiodigalactoside-Based Chemical Probes to Label Galectin-3. ChemBioChem 2009, 10, 1724-1733. [CrossRef] [PubMed]

23. Ballell, L.; van Scherpenzeel, M.; Buchalova, K.; Liskamp, R.M.J.; Pieters, R.J. A new chemical probe for the detection of the cancer-linked galectin-3. Org. Biomol. Chem. 2006, 4, 4387-4394. [CrossRef] [PubMed]

24. van Scherpenzeel, M.; van der Pot, M.; Arnusch, C.J.; Liskamp, R.M.J.; Pieters, R.J. Detection of galectin-3 by novel peptidic photoprobes. Bioorg. Med. Chem. Lett. 2007, 17, 376-378. [CrossRef] [PubMed]

25. Canales, A.; Mallagaray, A.; Berbís, M.A.; Navarro-Vázquez, A.; Domínguez, G.; Cañada, F.J.; André, S.; Gabius, H.-J.; Pérez-Castells, J.; Jiménez-Barbero, J. Lanthanide-Chelating Carbohydrate Conjugates Are Useful Tools to Characterize Carbohydrate Conformation in Solution and Sensitive Sensors to Detect Carbohydrate-Protein Interactions. J. Am. Chem. Soc. 2014, 136, 8011-8017. [CrossRef] [PubMed]

26. Park, Y.K.; Bold, B.; Lee, W.K.; Jeon, M.H.; An, K.H.; Jeong, S.Y.; Shim, Y.K. D-(+)-Galactose-Conjugated Single-Walled Carbon Nanotubes as New Chemical Probes for Electrochemical Biosensors for the Cancer Marker Galectin-3. Int. J. Mol. Sci. 2011, 12, 2946-2957. [CrossRef]

27. Rotariu, L.; Lagarde, F.; Jaffrezic-Renault, N.; Bala, C. Electrochemical biosensors for fast detection of food contaminants-trends and perspective. TrAC Trends Anal. Chem. 2016, 79, 80-87. [CrossRef]

28. Topkaya, S.N.; Azimzadeh, M.; Ozsoz, M. Electrochemical Biosensors for Cancer Biomarkers Detection: Recent Advances and Challenges. Electroanalysis 2016, 28, 1402-1419. [CrossRef]

29. Mehrvar, M.; Abdi, M. Recent Developments, Characteristics, and Potential Applications of Electrochemical Biosensors. Anal. Sci. 2004, 20, 1113-1126. [CrossRef]

30. Sadighbayan, D.; Sadighbayan, K.; Tohid-kia, M.R.; Khosroushahi, A.Y.; Hasanzadeh, M. Development of electrochemical biosensors for tumor marker determination towards cancer diagnosis: Recent progress. TrAC Trends Anal. Chem. 2019, 118, 73-88. [CrossRef]

31. Casas-Solvas, J.M.; Vargas-Berenguel, A.; Capitán-Vallvey, L.F.; Santoyo-González, F. Convenient methods for the synthesis of ferrocene-carbohydrate conjugates. Org. Lett. 2004, 6, 3687-3690. [CrossRef] [PubMed]

32. Casas-Solvas, J.M.; Ortiz-Salmerón, E.; Giménez-Martínez, J.J.; García-Fuentes, L.; Capitán-Vallvey, L.F.; Santoyo-González, F.; Vargas-Berenguel, A. Ferrocene-Carbohydrate Conjugates as Electrochemical Probes for Molecular Recognition Studies. Chem. Eur. J. 2009, 15, 710-725. [CrossRef] [PubMed]

33. Casas-Solvas, J.M.; Ortiz-Salmerón, E.; Fernández, I.; García-Fuentes, L.; Santoyo-González, F.; Vargas-Berenguel, A. Ferrocene- $\beta$-Cyclodextrin Conjugates: Synthesis, Supramolecular Behavior, and Use as Electrochemical Sensors. Chem. Eur. J. 2009, 15, 8146-8162. [CrossRef] [PubMed]

34. Casas-Solvas, J.M.; Ortiz-Salmerón, E.; García-Fuentes, L.; Vargas-Berenguel, A. Ferrocene-mannose conjugates as electrochemical molecular sensors for concanavalin A lectin. Org. Biomol. Chem. 2008, 6, 4230-4235. [CrossRef]

35. Martos-Maldonado, M.C.; Casas-Solvas, J.M.; Téllez-Sanz, R.; Mesa-Valle, C.; Quesada-Soriano, I.; García-Maroto, F.; Vargas-Berenguel, A.; García-Fuentes, L. Binding properties of ferrocene-glutathione conjugates as inhibitors and sensors for glutathione S-transferases. Biochimie 2012, 94, 541-550. [CrossRef]

36. Martos-Maldonado, M.C.; Thygesen, M.B.; Jensen, K.J.; Vargas-Berenguel, A. Gold-Ferrocene Glyco-Nanoparticles for High-Sensitivity Electrochemical Detection of Carbohydrate-Lectin Interactions. Eur. J. Org. Chem. 2013, 2013, 2793-2801. [CrossRef]

37. Martos-Maldonado, M.C.; Quesada-Soriano, I.; García-Maroto, F.; Vargas-Berenguel, A.; García-Fuentes, L. Ferrocene labelings as inhibitors and dual electrochemical sensors of human glutathione S-transferase P1-1. Bioorg. Med. Chem. Lett. 2012, 22, 7256-7260. [CrossRef]

38. Martos-Maldonado, M.C.; Casas-Solvas, J.M.; Quesada-Soriano, I.; García-Fuentes, L.; Vargas-Berenguel, A. Poly (amido amine)-Based Mannose-Glycodendrimers As Multielectron Redox Probes for Improving Lectin Sensing. Langmuir 2013, 29, 1318-1326. [CrossRef]

39. Martos-Maldonado, M.C.; Casas-Solvas, J.M.; Vargas-Berenguel, A.; García-Fuentes, L. Electrochemical Detection of Glutathione S-Transferase: An Important Enzyme in the Cell Protective Mechanism Against Oxidative Stress. Methods Mol. Biol. 2015, 1208, 123-138. [CrossRef]

40. Ahmad, N.; Gabius, H.-J.; André, S.; Kaltner, H.; Sabesan, S.; Roy, R.; Liu, B.; Macaluso, F.; Brewer, C.F. Galectin-3 Precipitates as a Pentamer with Synthetic Multivalent Carbohydrates and Forms Heterogeneous Cross-linked Complexes. J. Biol. Chem. 2004, 279, 10841-10847. [CrossRef] 
41. Böcker, S.; Elling, L. Binding Characteristics of Galectin-3 Fusion Proteins. Glycobiology 2017, $27,457-468$. [CrossRef]

42. Lepur, A.; Salomonsson, E.; Nilsson, U.J.; Leffler, H. Ligand Induced Galectin-3 Protein Self-association. J. Biol. Chem. 2012, 287, 21751-21756. [CrossRef] [PubMed]

43. Goodman, C.K.; Wolfenden, M.L.; Nangia-Makker, P.; Michel, A.K.; Raz, A.; Cloninger, M.J. Multivalent scaffolds induce galectin-3 aggregation into nanoparticles. Beilstein J. Org. Chem. 2014, 10, 1570-1577. [CrossRef] [PubMed]

44. Cagnoni, A.J.; Kovensky, J.; Uhrig, M.L. Design and synthesis of hydrolytically stable multivalent ligands bearing thiodigalactoside analogues for peanut lectin and human galectin-3 binding. J. Org. Chem. 2014, 79, 6456-6467. [CrossRef] [PubMed]

45. Aykaç, A.; Martos-Maldonado, M.C.; Casas-Solvas, J.M.; Quesada-Soriano, I.; García-Maroto, F.; García-Fuentes, L.; Vargas-Berenguel, A. $\beta$-Cyclodextrin-Bearing Gold Glyconanoparticles for the Development of Site Specific Drug Delivery Systems. Langmuir 2014, 30, 234-242. [CrossRef]

46. Lévy, R.; Thanh, N.T.K.; Doty, R.C.; Hussain, I.; Nichols, R.J.; Schiffrin, D.J.; Brust, M.; Fernig, D.G. Rational and Combinatorial Design of Peptide Capping Ligands for Gold Nanoparticles. J. Am. Chem. Soc. 2004, 126, 10076-10084. [CrossRef]

47. Turkevich, J.; Stevenson, P.C.; Hillier, J. The Formation of Colloidal Gold. J. Phys. Chem. 1953, 57, 670-673. [CrossRef]

48. Haiss, W.; Thanh, N.T.K.; Aveyard, J.; Fernig, D.G. Determination of size and concentration of gold nanoparticles from UV-Vis spectra. Anal. Chem. 2007, 79, 4215-4221. [CrossRef]

49. Weisbecker, C.S.; Merritt, M.V.; Whitesides, G.M. Molecular self-assembly of aliphatic thiols on gold colloids. Langmuir 1996, 12, 3763-3772. [CrossRef]

50. Quesada-Soriano, I.; Parker, L.J.; Primavera, A.; Casas-Solvas, J.M.; Vargas-Berenguel, A.; Barón, C.; Morton, C.J.; Mazzetti, A.P.; Lo Bello, M.; Parker, M.W.; et al. Influence of the H-site residue 108 on human glutathione transferase P1-1 ligand binding: Structure-thermodynamic relationships and thermal stability. Protein Sci. 2009, 18, 2454-2470. [CrossRef]

51. Malkoch, M.; Schleicher, K.; Drockenmuller, E.; Hawker, C.J.; Russell, T.P.; Wu, P.; Fokin, V.V. Structurally Diverse Dendritic Libraries: A Highly Efficient Functionalization Approach Using Click Chemistry. Macromolecules 2005, 38, 3663-3678. [CrossRef]

52. Thygesen, M.B.; Sauer, J.; Jensen, K.J. Chemoselective Capture of Glycans for Analysis on Gold Nanoparticles: Carbohydrate Oxime Tautomers Provide Functional Recognition by Proteins. Chem.-A Eur. J. 2009, 15, 1649-1660. [CrossRef] [PubMed]

53. Thygesen, M.B.; Jensen, K.J. Carbohydrate-Modified Gold Nanoparticles. In Carbohydrate Nanotechnology; Stine, K.J., Ed.; John Wiley \& Sons, Inc.: Hoboken, NJ, USA, 2015; pp. 79-98. [CrossRef]

54. Daniel, M.C.; Astruc, D. Gold Nanoparticles: Assembly, Supramolecular Chemistry, Quantum-Size-Related Properties, and Applications Toward Biology, Catalysis, and Nanotechnology. Chem. Rev. 2004, 104, $293-346$. [CrossRef] [PubMed]

55. Astruc, D. Ferrocenyl dendrimers: Multi-electron redox reagents and their applications. New J. Chem. 2011, 35, 764-772. [CrossRef]

56. Bard, A.J.; Faulkner, L.R. Electrochemical Methods: Fundamentals and Applications, 2nd ed.; Wiley: New York, NY, USA, 2001. [CrossRef]

57. Toone, E.J. Structure and energetics of protein-carbohydrate complexes. Curr. Opin. Struct. Biol. 1994, 4, 719-728. [CrossRef]

58. Dam, T.K.; Fred Brewer, C. Thermodynamic Studies of Lectin-Carbohydrate Interactions by Isothermal Titration Calorimetry. Chem. Rev. 2002, 102, 387-430. [CrossRef]

59. Dam, T.K.; Roy, R.; Das, S.K.; Oscarson, S.; Brewer, C.F. Binding of Multivalent Carbohydrates to Concanavalin A and Dioclea grandiflora Lectin. J. Biol. Chem. 2000, 275, 14223-14230. [CrossRef]

60. Hsieh, T.-J.; Lin, H.-Y.; Tu, Z.; Lin, T.-C.; Wu, S.-C.; Tseng, Y.-Y.; Liu, F.-T.; Hsu, S.-T.D.; Lin, C.-H. Dual thio-digalactoside-binding modes of human galectins as the structural basis for the design of potent and selective inhibitors. Sci. Rep. 2016, 6, 29457. [CrossRef] 
61. Marín, M.J.; Schofield, C.L.; Field, R.A.; Russell, D.A. Glyconanoparticles for colorimetric bioassays. Analyst 2015, 140, 59-70. [CrossRef]

62. Sánchez-Pomales, G.; Morris, T.A.; Falabella, J.B.; Tarlov, M.J.; Zangmeister, R.A. A lectin-based gold nanoparticle assay for probing glycosylation of glycoproteins. Biotechnol. Bioeng. 2012, 109, 2240-2249. [CrossRef] 